\title{
Cosmological bounces in spatially flat FRW spacetimes in metric $f(R)$ gravity
}

\author{
Niladri Paul ${ }^{\ddagger *}$, Saikat Nil Chakrabarty**, Kaushik Bhattacharya** $\dagger$ \\ ‡ IUCAA, Post Bag 4, Pune University Campus, Ganeshkhind, \\ Pune 411 007, India \\ ** Department of Physics, Indian Institute of Technology, \\ Kanpur 208016, India
}

September 12, 2014

\begin{abstract}
The present work analyzes the various conditions in which there can be a bouncing universe solution in $f(R)$ gravity. In the article an interesting method, to analyze the bouncing FRW solutions in a spatially flat universe using $f(R)$ gravity models using an effective Einstein frame description of the process, is presented. The analysis shows that a cosmological bounce in the $f(R)$ theory need not be described by an equivalent bounce in the Einstein frame description of the process where actually there may be no bounce at all. Nevertheless the Einstein frame description of the bouncing phenomena turns out to be immensely important as the dynamics of the bounce becomes amenable to logic based on general relativistic intuition. The theory of scalar cosmological perturbations in the bouncing universe models in $f(R)$ theories has also been worked out in the Einstein frame.
\end{abstract}

*Niladri Paul, did most of the work presented in this article when he was in the Physics Department of Indian Institute of Technology, Kanpur 208016, India.

${ }^{\dagger}$ E-mail: npaul@iucaa.ernet.in, snilch@iitk.ac.in, kaushikb@iitk.ac.in 


\section{Introduction}

If the BICEP2 [1] results are confirmed by future observational projects, as the Planck collaboration, then the scientific community can be fairly certain that some form of inflationary dynamics must be responsible for the growth of the very early universe. Inflation as a theory [2,3] solves various problems in cosmology, as the horizon problem, the entropy problem, the flatness problem and others. But standard inflationary theories have some inherent problems in them as the transPlanckian problem [4-8]. If inflation indeed happened in the very early phase of the universe then the earliest modes (which are entering our horizon now) which became superhorizon had wavelengths which are smaller than the Planck length at the beginning of inflation. If this happens it produces severe problem in the inflationary paradigm because then the calculations concerning the cosmological perturbations due to these modes, based on known laws of physics, breaks down. There are various propositions which tries to address these issues. Some authors have tried to modify the dispersion relation of the earliest modes [7] while others have proposed cosmological bounces in the general relativistic framework [9, 10] and some variations of it $11-18]$. Except these ideas there are proposals to bypass Einstein gravity and work with $f(R)$ gravity induced bouncing universe scenarios where the initial singularity in the big-bang model never arises 19 21]. Some interesting work regarding the stability of $f(R)$ theories was done in Ref. [22]. The $f(R)$ theory paradigm has also been used to provide a unified platform for inflation and late time acceleration of the universe [23]. Except the physics of the early universe $f(R)$ theories of gravity have been used in various other cosmological sectors as well, as in the problem of late time cosmic acceleration 24 27], the dark matter problem [28] and many others ${ }^{1}$. In the present article we will be dealing with higher derivative $f(R)$ theories of gravity and try to analyze the dynamics of the bouncing cosmologies for spatially flat FRW spacetimes.

We assume that in the very early phase of the universe, when the Ricci scalar may have attained a very high value (compared to some relevant preassigned scale), the dynamics of the universe was guided by an effective $f(R)$ theory of gravity which assisted a cosmological bounce. This naturally leads to a form of second order $f(R)$ which was used by Starobinsky [34 to address the issue of

\footnotetext{
${ }^{1}$ Most of the applications of $f(R)$ theories are appropriately summed up in the review articles in, Ref. 29], Ref. [30] and Ref. [31]. The mathematically inclined reader may also like Ref. 32 33 where some general features of $f(R)$ theory are nicely presented.
} 
inflation. In essence $f(R)$ theory enters our cosmological model in the same way as in Starobinsky's model of inflation but unlike Starobinsky's model where the modified gravity theory produces inflation in a universe devoid of matter, in our case the modified theory of gravity will ensure a non singular cosmological bounce in presence or absence of hydrodynamic matter. As in $f(R)$ theories of gravity the equations of motion of the metric involves higher order partial derivatives of the metric (compared to the second order theories in GR) we will often call $f(R)$ theories of gravity as higher derivative (HD) theories of gravity. In these HD theories of gravity the study of cosmological bounces become difficult because of the HD terms of the metric and consequently most of the times an analytic solution remains illusive. To tackle this problem we have proposed an alternative way of looking at these bouncing solutions in HD theories of gravity. Our method relies on the description of the bouncing phenomenon in a conformally related Einstein frame. Although the relations connecting the conformal frame, where an HD theory of gravity lives, and that of a conformally related Einstein frame were well studied for the case of Starobinsky inflation [34], the present analysis of the bouncing phenomena in the two frames requires a complete new way of interpreting the physics of cosmological evolution. This is because unlike the case of Starobinsky inflation here the conformally related frames will not portray the same bouncing phenomenon when the spatial curvature of the FRW spacetime vanishes. In this particular case if one assumes that there is a cosmological bounce in the HD theory then in the Einstein frame description of the events there is no equivalent bounce. As a consequence of this, one has to specify from the first which is the physical conformal frame and which is an auxiliary conformal frame used mainly to solve the problem.

The Einstein frame description of cosmological bounces, in presence of matter, requires a scalar field whose potential may not be bounded from below and a hydrodynamic fluid whose energy density and pressure is modulated by the scalar field strength. But these facts do not deter one from describing the bouncing phenomenon in the Einstein frame as because in the time period where the HD theory is supposed to be active the scalar field cannot roll down to its negative infinite potential depth. In our analysis of the bouncing phenomena in HD theories we will assume that the hydrodynamic matter (if present) will satisfy the weak energy conditions (WEC) at all times ${ }^{2}$. If WEC is violated then many of our

\footnotetext{
${ }^{2}$ The validity of the WEC naturally implies the validity of the null energy condition.
} 
predictions require to be modified. But fortunately it can be shown that in the HD theory of gravity one can have perfectly non-singular cosmological bounces for the FRW solutions with flat spatial hypersurfaces where the hydrodynamic matter always satisfies WEC. In the Einstein frame the energy conditions become intricate as there are two components of matter: one hydrodynamic and the other comprising of a scalar field whose potential is unbounded from below.

It has been shown in the article that the analysis of the bouncing phenomenon in the Einstein frame gives us many insights about the behavior of the cosmological evolution in the physical frame. Whether we will have a symmetrical bounce or an asymmetrical bounce in the HD theory can be predicted from the values of the scalar field and its first derivative with respect to the time variable in the Einstein frame at the time of bounce. Ultimately one has to use numerical methods to calculate the time evolution of the system but the numerical codes can be better handled in the Einstein frame. The article ends with an analysis of cosmological perturbations in the bouncing universe. The calculations are done in the Einstein frame. Once the evolution of the perturbations are known in the Einstein frame one can map the solutions back to the conformal frame where the HD theory lives.

The material in the article is presented in the following way. The next section describes the very general framework of HD theories of $f(R)$ gravity and the bouncing conditions for spatially flat FRW spacetimes. In section 3 we present the topic of relativity of conformally connected frames which deals with the description of the cosmological bounce phenomenon as observed, in the conformal frame where the HD theory lives and in the related Einstein frame. Section 4 describes the bouncing phenomenon in the HD theory where we present the numerical results of actual calculations regarding cosmological bounce in FRW spacetimes with flat spatial sections in presence of radiation. The next section 5 gives a detailed description of the methods employed to solve the dynamical equations in the Einstein frame. Section 6 addresses the issue of scalar cosmological perturbations in the bouncing universe models as observed from the Einstein frame. The article ends with a discussion and summary of the results presented in it. 


\section{The cosmological model}

In this article we will mainly focus on bouncing cosmologies in the spatially flat FRW universe. In GR one cannot have a cosmological bounce in the flat FRW universe when matter obeys standard energy conditions [35]. On the other hand in higher derivative gravity one can have bouncing cosmologies in flat FRW case, as reported in Refs. [19,20]. In this article we will focus on the forms of $f(R)$ as given by

$$
f(R)=R+\alpha R^{n}
$$

where $\alpha$ is a real number and $n$ is an integer greater than one. In Starobinsky's model of inflation $n=2$ and $\alpha>0$. The major portion of this article will be dealing with the case where $n=2$ but unlike Starobinsky's model one requires $\alpha<0$ for a cosmological bounce. This form of $f(R)$ will modify the early stages of evolution of the universe and can only produce a cosmological bounce in presence of matter. The correction term in $f(R)$ which is $\alpha R^{n}$ may have originated from quantum corrections [36 to the standard GR action, in the early universe. Before going into the formalism of the HD theory of gravity predicted by the quadratic form of $f(R)$ the reader must be reminded that the quadratic theory of gravity with a negative $\alpha$ is by itself not stable as one cannot ensure $f^{\prime}(R)>0$ throughout the cosmic evolution. This problem can be avoided in certain cases, where the instability can be avoided by suitably choosing the parameter $\alpha$, or by generalizing the form of $f(R)$ in such a way that the instabilities are taken care of. As this

topic requires a detailed discussion we present it in a later subsection 2.3, after presenting the basic formalism of HD theories.

Following the notation of Ref. [30], the fundamental equation of $f(R)$ theory which corresponds to the Einstein's equation in GR, is

$$
\begin{aligned}
G_{\mu \nu} \equiv R_{\mu \nu}-\frac{1}{2} g_{\mu \nu} R & =\frac{\kappa T_{\mu \nu}}{f^{\prime}(R)}+g_{\mu \nu} \frac{\left[f(R)-R f^{\prime}(R)\right]}{2 f^{\prime}(R)} \\
& +\frac{\nabla_{\mu} \nabla_{\nu} f^{\prime}(R)-g_{\mu \nu} \square f^{\prime}(R)}{f^{\prime}(R)},
\end{aligned}
$$

where the prime denotes a derivative with respect to the Ricci scalar $R, T_{\mu \nu}$ is the energy momentum tensor of the fluid which permeates the spacetime, $\nabla_{\mu}$ is the covariant derivative and $\square \equiv \nabla^{\mu} \nabla_{\mu}$. Using the FRW line element

$$
d s^{2}=-d t^{2}+a^{2}(t)\left[\frac{d r^{2}}{1-k r^{2}}+r^{2}\left(d \theta^{2}+\sin ^{2} \theta d \phi^{2}\right)\right],
$$


and the energy momentum tensor of a perfect fluid

$$
T_{\mu \nu}=(\rho+P) u_{\mu} u_{\nu}+P g_{\mu \nu}
$$

one can write the various components of Eq. (2) in the form

$$
\begin{aligned}
3\left(\frac{k}{a^{2}}+H^{2}\right) & =\frac{\kappa}{f^{\prime}(R)}\left(\rho+\rho_{\mathrm{eff}}\right), \\
\frac{k}{a^{2}}+3 H^{2}+2 \dot{H} & =\frac{-\kappa}{f^{\prime}(R)}\left(P+P_{\mathrm{eff}}\right),
\end{aligned}
$$

where $H$ is the conventional Hubble parameter defined as $H \equiv \dot{a} / a$ and $k$ stands for the constant specifying the curvature of the 3-dimensional spatial hypersurface. The dot specifies a derivative with respect to cosmological time $t$ and $\rho_{\text {eff }}$ and $P_{\text {eff }}$ are defined as:

$$
\begin{aligned}
\rho_{\mathrm{eff}} & \equiv \frac{R f^{\prime}-f}{2 \kappa}-\frac{3 H \dot{R} f^{\prime \prime}(R)}{\kappa}, \\
P_{\text {eff }} & \equiv \frac{\dot{R}^{2} f^{\prime \prime \prime}+2 H \dot{R} f^{\prime \prime}+\ddot{R} f^{\prime \prime}}{\kappa}-\frac{R f^{\prime}-f}{2 \kappa},
\end{aligned}
$$

which are like effective energy-density and pressure due to the curvature effects in contrast to the conventional $\rho$ and $P$ in Eq. (4) which are the energy-density and pressure of the fluid present in the FRW spacetime. In this article we assume the fluid to be barotropic so that its equation of state is

$$
P=\omega \rho,
$$

where $\omega$ is zero for dust and one-third for radiation. It must be noted that $u_{\mu}$ in Eq. (4) is the four-velocity of a fluid element and $u_{\mu} u^{\mu}=-1$.

\subsection{General bouncing conditions}

In this section we will primarily focus on the bouncing conditions in the early universe keeping an eye on the energy conditions which are followed by the matter sector. Some important results regarding the bouncing conditions in FRW cosmologies were reported in Ref. 21] where the authors very briefly inferred about the possibility of cosmological bounces in flat FRW cosmologies. In this article we will solely focus on cosmological bounces in flat FRW models and discuss various interesting phenomena related to these bounces. Writing the time 
coordinate during the bounce as $t=t_{b}$ and the Hubble parameter at bounce as $H_{b}=H\left(t_{b}\right)$, the conditions for cosmological bounce are

$$
H_{b}=0, \text { and } \dot{H}_{b}>0 .
$$

Henceforth the subscript $b$ on any variable (which varies with time) will specify its value at the time of bounce. In this convention one can write the vanishing condition of the Hubble parameter during the bounce as

$$
3 \frac{k}{a_{b}^{2}}=\frac{\kappa}{f_{b}^{\prime}}\left[\rho_{b}+\frac{R_{b} f_{b}^{\prime}-f_{b}}{2 \kappa}\right] .
$$

The positivity of the time derivative of $H$ during bounce becomes

$$
2 \dot{H}_{b}=-\frac{\kappa}{f_{b}^{\prime}}\left[P_{b}+\frac{\dot{R}_{b}^{2} f_{b}^{\prime \prime \prime}+\ddot{R}_{b} f_{b}^{\prime \prime}}{\kappa}-\frac{R_{b} f_{b}^{\prime}-f_{b}}{2 \kappa}\right]-\frac{k}{a_{b}^{2}}>0,
$$

which can also be written as

$$
\kappa\left(P_{b}+\rho_{b}\right)+\dot{R}_{b}^{2} f_{b}^{\prime \prime \prime}+\ddot{R}_{b} f_{b}^{\prime \prime}-2 \frac{k f_{b}^{\prime}}{a_{b}^{2}}<0 .
$$

If one assumes the hydrodynamic matter in the cosmological background satisfies the standard weak energy condition as applied in GR:

$$
\rho \geq 0, \quad \rho+P \geq 0,
$$

then Eq. 12 implies

$$
\dot{R}_{b}^{2} f_{b}^{\prime \prime \prime}+\ddot{R}_{b} f_{b}^{\prime \prime}-2 \frac{k f_{b}^{\prime}}{a_{b}^{2}}<0 .
$$

Using the form of $f(R)$ as given in Eq. (1), the above equations predict that during a bounce the following conditions must be fulfilled:

$$
\rho_{b}+\frac{(n-1) \alpha R_{b}^{n}}{2 \kappa}=0,
$$

for the spatially flat case, $k=0$. When spatial curvature is zero, $R_{b}=6 \dot{H}_{b}$, and in such a case one can have a bouncing universe in the quadratic gravity model, as specified in Eq. (1), when $\rho_{b} \neq 0$. Without matter there is no bounce with the form of $f(R)$ chosen in Eq. (1) in the spatially flat FRW model. More over as $n>1$ it can be seen from Eq. 15 that $\alpha<0$. 
For the sake of completeness we also briefly state that there can be cosmological models in $f(R)$ theory where the presence of matter is not absolutely important for the bouncing phenomenon. If one takes

$$
f(R)=R+\beta R^{2}+\gamma R^{3},
$$

where $\beta$ and $\gamma$ are real numbers it can be shown that this form of $f(R)$ is capable of producing cosmological bounce without matter. In particular for the flat FRW spacetime in absence of any matter the bouncing condition becomes

$$
\left(R_{b} f_{b}^{\prime}-f_{b}\right)=0
$$

predicting that

$$
R_{b}=-\frac{\beta}{2 \gamma} .
$$

As $R_{b}$ is positive definite for matter-less bounce in the flat FRW universe, one has to assume that $\gamma$ and $\beta$ must have different signs. In the present case if one demands that $f^{\prime}(R)>0$, such that the effective gravitational constant remains positive as discussed in Ref. [37], the coefficients $\beta$ and $\gamma$ must satisfy the following inequality

$$
0<\beta^{2} \leq 3 \gamma
$$

From Eq. 180 it was seen that $\beta$ and $\gamma$ should have opposite sign whereas from the above equation it can be uniquely said that for a bouncing solution $\gamma>0$ and $\beta<0$. With the form of $f(R)$ as given in Eq. (16) one can easily verify that the third order gravitational action arising from it can also support a cosmological bounce in flat FRW universe in the presence of matter.

The above discussion on the possibility of bounces, in various $f(R)$ theories, was based on fact that the Hubble parameter must vanish at $t=t_{b}$ as stated in Eq. (11). We did not discuss about the second condition, the positivity of the rate of change of the Hubble parameter at $t=t_{b}$, as given in Eq. (14). The main reason for doing so is that these two conditions are independent. For a spatially flat universe, the first condition of bounce in Eq. (11) only depends on the value of $R_{b} 3^{3}$ whereas the second condition in Eq. 14 depends only on $R_{b}, \dot{R}_{b}$ and $\ddot{R}_{b}$. But all of these parameters are not independent of each other. It can be

\footnotetext{
${ }^{3}$ In this case $\rho_{b}$ is fixed when one specifies $R_{b}$.
} 
easily shown that the system of equations governing the dynamics of a flat FRW spacetime around a cosmological bounce, in presence of barotropic matter, can be determined if one knows the values of just two independent parameters $R_{b}$ and $\dot{R}_{b}$. Whereas for a similar bounce in absence of matter the value of $R_{b}$ is a constant as in Eq. (18), and the only independent parameter left is that of $\dot{R}_{b}$. The above discussion shows that the first bouncing condition, in Eq. (11), only depends upon the value of $R_{b}$ and the second bouncing condition, in Eq. (14), essentially only depends upon the values of $R_{b}$ and $\dot{R}_{b} 4^{4}$. Consequently, if in a cosmological model the first bouncing condition is satisfied for some value of $R_{b}$ the second bouncing condition can also be satisfied by suitably choosing some value of $\dot{R}_{b}$.

We have seen that some form of $f(R)$ can only produce a cosmological bounce in presence of matter and some other form of $f(R)$ is capable of producing a cosmological bounce in absence of matter. A natural question arises at this point, whether there can be any quantitative reasoning based on which one can differentiate these two forms of $f(R)$. We partially answer this in the next section where we present a brief analysis about the form of $f(R)$ which can give rise to a cosmological bounce, in absence of matter, in a flat FRW universe. The answer is partial because the form of $f(R)$ which can produce cosmological bounce in absence of matter may also be capable of producing a cosmological bounce in the presence of matter.

\subsection{Conditions for matter-less cosmological bounce in the flat FRW universe}

In the most general case one can state the following facts about a bouncing universe. Given an $f(R)$ such that $\left(R f^{\prime}-f\right)$ has at least a positive root, then there always exists a matter-less bounce in the spatially flat FRW metric. Further, if $\lim _{R \rightarrow 0} f(R)=0$, then the above condition implies that $f^{\prime \prime \prime}(R)$ cannot be identically zero for a matter-less bounce. The proof of the above statements follow. In a matter-less universe for $k=0$, if the Hubble parameter vanishes at any particular value of the Ricci scalar then one must have

$$
R f^{\prime}-f=0 \text {. }
$$

\footnotetext{
${ }^{4}$ As $\ddot{R}_{b}$ is is fixed once the values of $R_{b}$ and $\dot{R}_{b}$ are known.
} 
More over in our case for $k=0$, the value of the Ricci scalar when the Hubble parameter vanishes is given by

$$
R=6 \dot{H} .
$$

If for a particular $f(R)$ the function $R f^{\prime}-f$ has at least one positive root $R=R_{b}$ then it implies $H=0$ at $R=R_{b}$. More over from the above equation one can see that $\dot{H}$ remains positive when $R=R_{b}$. The last two conditions, on the Hubble parameter and its time derivative, specifies that there is a cosmological bounce at $R=R_{b}$.

If one also assumes that $\lim _{R \rightarrow 0} f(R)=0$ (eliminating any $f(R)$ with a cosmological constant like term in it) then one can write

$$
R f^{\prime}-f=R\left(R-R_{b}\right) g(R)
$$

where $g(R)$ is nonsingular at $R=0$ and $R=R_{b}$. Differentiating the last equation one gets

$$
R f^{\prime \prime}=\left(2 R-R_{b}\right) g+R\left(R-R_{b}\right) g^{\prime} .
$$

Differentiating again, and using the above equation, one gets

$$
R^{2} f^{\prime \prime \prime}=R_{b} g+R\left(3 R-R_{b}\right) g^{\prime}+R^{2}\left(R-R_{b}\right) g^{\prime \prime} .
$$

Now we make the proposition that a necessary condition for matter-less bounce is that $R^{2} f^{\prime \prime \prime}$ is not identically zero for the particular $f(R)$. To prove this, we assume the contrary, i.e. $R^{2} f^{\prime \prime \prime}$ is identically zero for the particular form of $f(R)$. In the present case then the function $g(R)$ must be the solution of the differential equation

$$
R^{2}\left(R-R_{b}\right) g^{\prime \prime}+R\left(3 R-R_{b}\right) g^{\prime}+R_{b} g=0 .
$$

This equation has an exact solution of the form

$$
g(R)=\frac{C_{1}}{R\left(R-R_{b}\right)}+\frac{C_{2} R}{2\left(R-R_{b}\right)},
$$

where $C_{1}$ and $C_{2}$ are arbitrary integration constants. The solution diverges at $R=R_{b}$ for all arbitrary constant values of $C_{1}$ and $C_{2}$. This implies that in the most general case $g(R)$ is singular at $R=R_{b}$, defying our assumption that $g(R)$ is non singular at $R_{b}$. Consequently the assumption that $R^{2} f^{\prime \prime \prime}$ is identically zero cannot be true. The last statement also implies that $f^{\prime \prime \prime}$ cannot be identically zero in an interval of $R$ which contains the bouncing phase. 
Before ending this section we must remind the reader that the possibility of a matter-less bounce in the flat FRW universe, in metric $f(R)$ gravity, does not exclude the possibility of a cosmological bounce in presence of matter satisfying the energy conditions as given in Eq. (13). On the other hand there can be situations where one cannot have a cosmological bounce in HD gravity in the absence of matter as discussed in the case of quadratic gravity.

\subsection{Stability analysis in quadratic gravity with negative}

The form of $f(R)$ in Eq. (1) for $\alpha<0$ suggests that $f^{\prime}(R)$ may not be positive for all values of $R$. Particularly, if $n=2$ then $f^{\prime}(R)=1+2 \alpha R$ which implies that for $R>\Lambda=1 /(2|\alpha|), f^{\prime}(R)<0$ making the theory unstable as the effective gravitational constant becomes negative. During the bouncing period the value of $R$ remains below the instability scale, $\Lambda$, as can be verified from the plot of the logarithm of $f^{\prime}(R)$ (which is proportional to the scalar field $\varphi_{0}$ in the Einstein frame, introduced in a later section), in Fig. (7). The instability in the theory can show up near the end stages of the bouncing period where $R$ can reach the instability scale. This issue can be addressed in broadly two different ways.

1. One may choose the value of $\alpha$ in a particular way so that the instability scale $\Lambda$ remains less than the value of the Ricci scalar at the present epoch of cosmic expansion. In such a case $f^{\prime}(R)>0$ throughout the cosmic evolution if the initial value of the Ricci scalar during the contracting phase is less than $\Lambda$. This method of evading the instability may be practical but not consistent as the instability may show up in the future unless the theory of gravity itself changes.

2. If $\Lambda$ has a high value then the form of quadratic $f(R)$ can certainly become unstable much earlier in our cosmic history. In this case if one wants to study the cosmological bounce in the quadratic theory then one has to stabilize the theory. To make the theory stable one can enlarge the form of $f(R)$, by adding suitable terms to it, in such a way that the new terms in $f(R)$ makes the theory stable and at the same time does not change the nature of the cosmological bounce as predicted from the simple form of

quadratic gravity as given in Eq. (1). Consequently one may work with the quadratic form of $f(R)$ to investigate the time period near the cosmological 
bounce. To execute the above plan one may generalize the form of $f(R)$ as:

$$
f(R)=R+\alpha R^{2}+G(R),
$$

where $G(R)$ is a function of $R$ which is negligible during the bouncing period. A probable form of $G(R)$ can be

$$
G(R)=\sum_{n} \xi_{n}\left(R-R_{c}\right)^{n},
$$

where $\xi_{n}$ s are constants, $R_{c}$ is another constant whose dimension is that of the Ricci scalar and $n(\neq 0)$ is an integer which can take both positive and negative values.

There can be a multiple number of ways in which one can choose a $G(R)$. In this article we give a simple form of $G(R)$ which can reasonably stabilize the quadratic HD theory. If one sets $n=3, R_{c}=0$ and $\xi_{3}>0$ and all other $\xi_{n}=0$ for $n \neq 3$, then $f(R)$ becomes a cubic polynomial of $R$ and in such a case if $3 \xi_{3}>\alpha^{2}$ one can assure that $f^{\prime}(R)>0$ for all values of $R$, as shown in Eq. (19). By choosing suitable values of $|\alpha|$ one can easily satisfy the condition

$$
G(R) \equiv \xi_{3} R^{3}<R+\alpha R^{2},
$$

if $R$ remains lower than $\Lambda$. When $R \rightarrow \Lambda$ the cubic term starts to contribute in the expression of $f(R)$ and in this limit the energy-density of the hydrodynamic matter, which supports the cosmological bounce tends to vanish due to cosmic expansion. In general near the bounce the above form of $G(R)$ is two orders of magnitude less than the pure quadratic term in the enlarged expression of $f(R)$ if $\alpha \sim 10^{11-12} M_{P}^{-2}$ and $R<\Lambda$. Here $M_{P} \sim 10^{19} \mathrm{GeV}$ is the Planck mass. Consequently, in this case the cubic term can be neglected during the brief bouncing phase and one may treat the cosmological bounce problem in the quadratic gravity paradigm.

The above discussion on the stabilization of the theory of gravity near the bouncing point shows that the HD quadratic gravity theory is more like an effective theory of gravity. Near the bouncing point only the terms up to quadratic order in the Ricci scalar contributes in the gravitational action whereas away from the bounce other terms in the action (of the general theory of gravity) becomes active. As the effect of the function $G(R)$ can be neglected during cosmological 


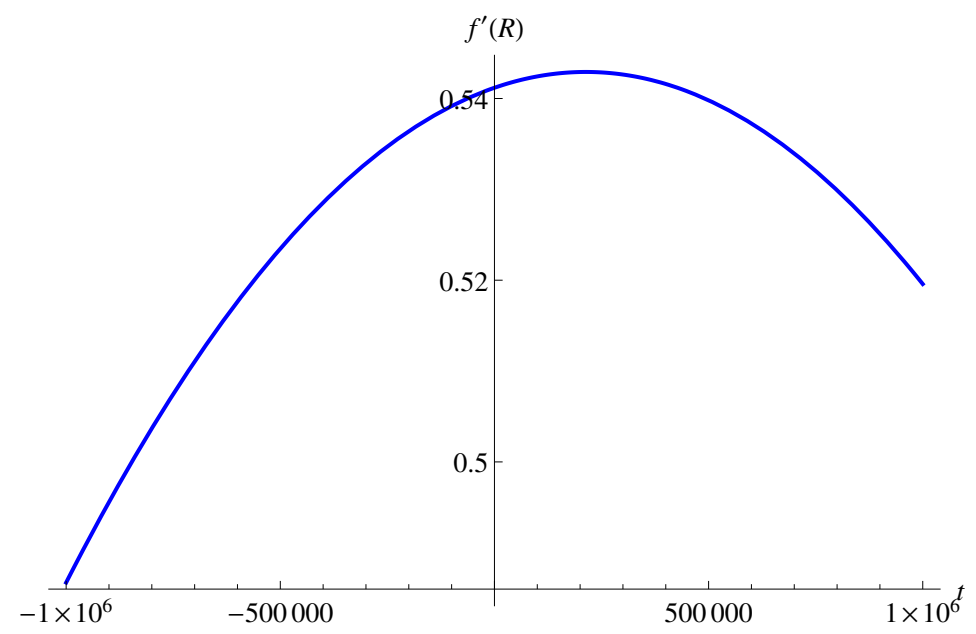

Figure 1: Plot of $F(R)=f^{\prime}(R)$ with respect to time in the Jordan frame in a radiation induced bounce in the quadratic gravity model. Here Ricci scalar and cosmic time are expressed in Planck units. The details of the model is presented in subsection 5 where the topic of radiation induced bounce is presented.

bounce predicted from the quadratic $f(R)$ in presence of matter we will henceforth not discuss the effects of $G(R)$ in the bouncing phenomenon. In this article we do not claim to give a full cosmological evolution of the early universe to the present universe. The main aim of this article is to describe the bouncing phase using a quadratic gravity theory where one may require the $G(R)$ function to avoid the intrinsic instability related to the quadratic HD theory.

In Fig. 1 we present a specimen plot of $f^{\prime}(R)$ with respect to time, during the radiation induced bounce in the quadratic gravity model (disregarding the effects of $G(R))$ and show that $f^{\prime}(R)$ remains positive during the bouncing regime. If $G(R)$ is included then the nature of the curve will change during the end phases of the bouncing period. The particulars of the model which produces the features of the plot will be explained in section 3 where we discuss the detailed analysis of the bouncing mechanism.

\section{Relativity of conformally connected frames}

In this section we will present a detailed analysis of the bounce mechanism in HD gravity. As HD gravity theories in the presence of matter is a difficult theory to solve analytically we will take recourse to a method which relies on relativity of 
conformally connected frames. The HD theory is defined in a frame which is often called the matter frame 24] or the Jordan frame. As one can always cast a metric $f(R)$ theory of gravity in the Jordan frame, where the theory of gravity looks like a Brans-Dicke theory with the Brans-Dicke parameter $\omega_{0}=0$ [30] without doing any conformal transformation, we will mostly call the conformal frame of the HD theory to be the Jordan frame.

Once the HD gravity theory is set in the matter or Jordan frame one can make a conformal transformation from the Jordan frame to the Einstein frame where the corresponding theory will be described by the Einstein's equation in GR and the dynamics of a scalar field which is minimally coupled to gravity and non-minimally coupled to matter. It is a general belief that the description of gravitational physics in the Jordan frame is equivalent to that in the Einstein frame. In a previous work in Ref. 38 the authors, who have studied the nature of gravitational waves in both conformal frames, have shown that this belief need not be always true. In Ref. [39] the authors specifically mentioned that the cosmological evolution in Jordan frame and the Einstein frame can be different in $f(R)$ theories. In this article we will observe a clear case where the description of cosmology in these two conformal frames are different. If there is a cosmological bounce in the flat FRW universe in the Jordan frame there cannot be any analogous bounce in the Einstein frame.

As the two conformal frames in our case do not predict equivalent bouncing cosmologies, we have to choose the frame which is physical, as discussed in [39]. In our specific case as the HD theory is initially presented in the Jordan frame we will take the Jordan frame to be the physical frame. The Einstein frame serves as an auxiliary frame in which the calculations are done for simplicity. This distinction between the physical frame and the auxiliary frame was previously discussed in the section regarding conformal transformations in Ref. [31]. The conformal transformations from the matter frame to the Einstein frame have been studied earlier [31, 33, 37, 40].

\subsection{Einstein frame description of higher-derivative grav- ity}

Starting from the dynamical equation of motion of the gravitational field in the higher-derivative $f(R)$ theory as given in Eq. (2) one can apply a conformal 
transformation as

$$
\tilde{g}_{\mu \nu}=F(R) g_{\mu \nu}
$$

where

$$
F(R) \equiv \frac{d f(R)}{d R},
$$

and obtain an effective equation of gravitational dynamics in the Einstein frame as

$$
\tilde{R}_{\nu}^{\mu}-\frac{1}{2} \delta_{\nu}^{\mu} \tilde{R}=\kappa \tilde{S}_{\nu}^{\mu}
$$

The energy-momentum tensor is given as

$$
\tilde{S}_{\nu}^{\mu}=\tilde{T}_{\nu}^{\mu}+\tilde{\mathcal{T}}_{\nu}^{\mu}
$$

where $\tilde{T}_{\nu}^{\mu}$ is the energy-momentum tensor of a real scalar field $\varphi_{0}$ defined as

$$
\varphi_{0} \equiv \sqrt{\frac{3}{2 \kappa}} \ln F
$$

Here the subscript 0 in $\varphi_{0}$ specifies that the scalar field controls the background cosmological evolution in the Einstein frame. Later we will deal with perturbations on this background, $\delta \varphi$, to study the evolution of the cosmological perturbations in a bouncing universe. For the particular model of quadratic $f(R)$ one can always satisfy the condition $F>0$ near the bounce point and so the above definition of the scalar field will pose no problem. The potential of the scalar field in the Einstein frame is given by

$$
V\left(\varphi_{0}\right)=\frac{R F-f}{2 \kappa F^{2}}
$$

The $\varphi_{0}$ dependence of $V$ is obtained by expressing $R$ as a function of $\varphi_{0}$ using Eq. (32) and the definition of $F(R)$. For an example, if one assumes $n=2$ in Eq. (1), then the expression of $V\left(\varphi_{0}\right)$ is of the following form:

$$
V\left(\varphi_{0}\right)=\frac{1}{8 \kappa \alpha}\left(1-e^{-\sqrt{2 \kappa / 3} \varphi_{0}}\right)^{2}
$$

whose plot is shown in Fig. 2. The second term in $\tilde{S}_{\nu}^{\mu}$ is $\tilde{\mathcal{T}}_{\nu}^{\mu}$ and it is related to the actual energy-momentum tensor of the fluid as given in Eq. (4) via

$$
\tilde{\mathcal{T}}_{\nu}^{\mu}=\frac{T_{\nu}^{\mu}}{F^{2}}
$$




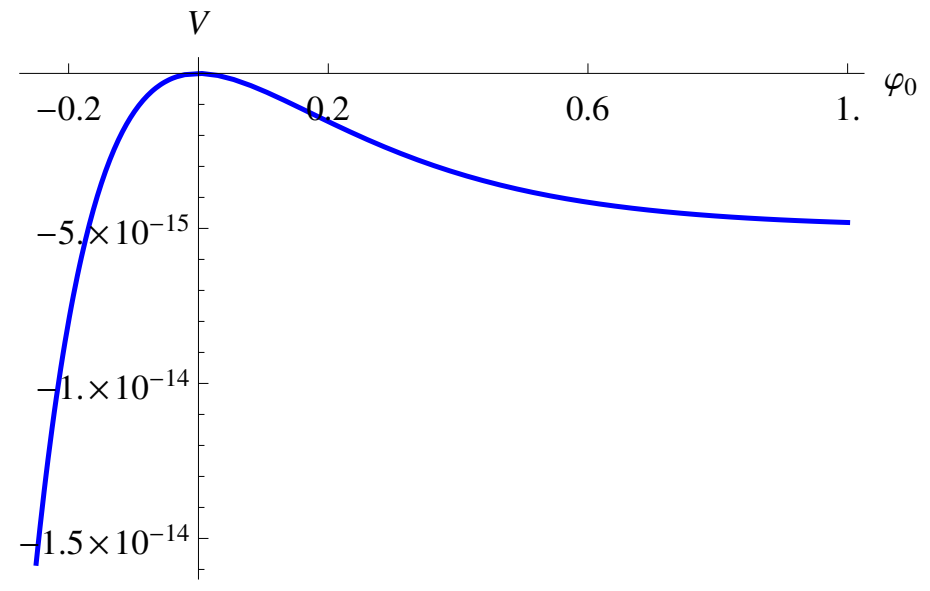

Figure 2: Figure showing the nature of the scalar field potential in the Einstein frame where $f(R)=R+\alpha R^{2}$. In the above figure the field and its potential both are expressed in Planck units (where the Planck mass is set as unity) in which $\alpha=-10^{12}$. The reason for such a magnitude of $\alpha$ is explained in the text below.

Using the transformed metric $\tilde{g}_{\alpha \beta}$ to lower the contravariant index in $\tilde{\mathcal{T}}_{\nu}^{\mu}$ one can rewrite the above equation in the following form

$$
\tilde{\mathcal{T}}_{\mu \nu}=(\tilde{\rho}+\tilde{P}) \tilde{u}_{\mu} \tilde{u}_{\nu}+\tilde{P} \tilde{g}_{\mu \nu}
$$

where the new energy-density, pressure and the 4-velocity of the hydrodynamic fluid element are

$$
\tilde{\rho}=\frac{\rho}{F^{2}}, \tilde{P}=\frac{P}{F^{2}}, \tilde{u}_{\mu} \equiv \sqrt{F} u_{\mu}
$$

The new 4 -vectors $\tilde{u}_{\mu}$ are defined in such a way that $\tilde{g}^{\mu \nu} \tilde{u}_{\mu} \tilde{u}_{\nu}=-1$. It is interesting to note that if the equation of state of the fluid in the higher-derivative description was $P=\omega \rho$ then in the Einstein frame the equation of state of the fluid remains the same.

One can now interpret Eq. (30) as the equivalent FRW description, in GR, of the HD gravitational theory where the new line element written in terms of the conformally transformed metric $\tilde{g}_{\mu \nu}$ looks like

$$
d \tilde{s}^{2}=-d \tilde{t}^{2}+\tilde{a}^{2}(\tilde{t})\left[\frac{d r^{2}}{1-k r^{2}}+r^{2}\left(d \theta^{2}+\sin ^{2} \theta d \varphi_{0}^{2}\right)\right],
$$

where

$$
d \tilde{t}=\sqrt{F(R)} d t, \quad \tilde{a}(t)=\sqrt{F(R)} a(t) .
$$


The above equations provide us the redefined time in the Einstein frame corresponding to the time variable appearing in the original FRW metric in Eq. (3) in the higher-derivative theory, as

$$
\tilde{t}=\int_{t_{b}}^{t} \sqrt{F(R)} d t,
$$

where the lower integration limit is set at the bouncing time in the Jordan frame. The relation between $t$ and $\tilde{t}$ shows that whatever be the value of $t_{b}$, the time corresponding to it in the Einstein frame, $\tilde{t}=0$.

The HD effects can be important in the early universe when the Hubble parameter was big, $H \sim 10^{12-13} \mathrm{GeV}$. For this value of the Hubble parameter one obtains $R \sim 10^{26} \mathrm{GeV}^{2}$ assuming a very slow change of $H$ with time. If the HD correction $\alpha R^{2}$ becomes effective near about this time then $|\alpha| \sim 1 / R$ yielding $|\alpha| \sim 10^{-26} \mathrm{GeV}^{-2}=10^{12} M_{P}^{-2}$. The relationship of quadratic gravity, giving rise to a matter induced cosmological bounce, and a more general cubic form of $f(R)$ containing more ingredients in it was discussed in subsection 2.3 . The chosen value of $\alpha$ in our case is such that one may use the quadratic form of HD gravity theory near the bounce.

The relation between the Hubble parameter, $H$, appearing in the higherderivative gravity theory and the effective Einstein frame Hubble parameter $\tilde{H}$, defined as $\tilde{a}^{\prime} / \tilde{a}$, is

$$
H=\sqrt{F}\left(\tilde{H}-\sqrt{\frac{\kappa}{6}} \varphi_{0}^{\prime}\right)
$$

where the prime now stands for $d / d \tilde{t}^{5}$. The Einstein frame Hubble parameter satisfies the following Friedman equation

$$
\tilde{H}^{2}+\frac{k}{\tilde{a}^{2}}=\frac{\kappa}{3}\left(\rho_{\varphi_{0}}+\tilde{\rho}\right)
$$

where

$$
\rho_{\varphi_{0}}=\frac{1}{2} \varphi_{0}^{\prime 2}+V\left(\varphi_{0}\right)
$$

\footnotetext{
${ }^{5}$ At this point we must alert the reader that we will use a superscript prime to symbolize three different quantities in this article. In subsection 2.1 the prime stood for a derivative with respect to the Ricci scalar. In this section the prime stands for a derivative with respect to the time variable in the Einstein frame. In the section 6 the prime will stand for a derivative with respect to the conformal time.
} 
and the expression for $\tilde{\rho}$ is given in Eq. (37). The other relevant equation for our case is

$$
\tilde{H}^{\prime}=\frac{k}{\tilde{a}^{2}}-\frac{\kappa}{2}\left[\varphi_{0}^{\prime 2}+(1+\omega) \tilde{\rho}\right]
$$

where $\omega$ appears in the equation of state of the fluid whose energy-momentum tensor is given in Eq. (36). Taking the trace of Einstein equation one gets the time evolution equation for the scalar field $\varphi_{0}$ as:

$$
\varphi_{0}^{\prime \prime}+3 \tilde{H} \varphi_{0}^{\prime}+\frac{d V}{d \varphi_{0}}=\sqrt{\frac{\kappa}{6}}(1-3 \omega) \tilde{\rho} .
$$

The above equation shows that time development of the scalar field depends upon the presence of the fluid energy-density. There is a coupling of matter and the scalar field in the Einstein frame. Only in the case of radiation does the right hand side of the above equation becomes zero and the scalar field can evolve in the conventional way. A radiation bath does not affect the scalar field dynamics due to conformal symmetry. The evolution of the energy density in the Einstein frame follows:

$$
\tilde{\rho}^{\prime}+\sqrt{\frac{\kappa}{6}}(1-3 \omega) \tilde{\rho} \varphi_{0}^{\prime}+3 \tilde{H} \tilde{\rho}(1+\omega)=0 .
$$

The set set of equations, Eq. (41), Eq. (43), Eq. (44) and Eq. (45), are the main equations which dictate the behavior of the system in the Einstein frame.

From the dynamical equations of the cosmological model presented in the Einstein frame it may seem that one must require the values of a plethora of variables at the bounce time to run the system analytically or numerically. But a closer inspection reveals that in the case of the spatially flat FRW spacetime the values of just two quantities, at the bouncing time, are enough to specify the past and future of the universe around the bouncing point. In this article we will take these two quantities to be $\varphi_{0}$ and $\varphi_{0}^{\prime}$ at $\tilde{t}=0$.

Next we state an interesting relationship of the bounce in the Jordan frame and its analogous behavior in the Einstein frame.

\subsection{On the absence of bounce in the Einstein frame}

A cosmological bounce in the flat FRW space in metric $f(R)$ gravity theory in the Jordan frame will never have an analogous cosmological bounce in the conformally related Einstein frame if matter satisfies the condition $\rho+P \geq 0$. The statement 
holds even in the absence of matter. The proof of the above statements follows. If there is a cosmological bounce in the Jordan frame for some particular form of $f(R)$ in the flat FRW universe then at the time of bounce $t=t_{b}$ one must have $H_{b}=0$ corresponding to which one gets $\tilde{H}(0)=\sqrt{\kappa / 6} \varphi_{0}^{\prime}(0)$ from Eq. 40 in the conformally related Einstein frame. The last relation shows that $\tilde{H}(0)$ need not be zero at $\tilde{t}=0$ which corresponds to the bouncing time in the Jordan frame. More over from Eq. 433) one one can see that $\tilde{H}^{\prime}(0)=-\frac{\kappa}{2}\left(\varphi_{0}^{\prime 2}+\tilde{\rho}+\tilde{P}\right)<0$ if matter respects the energy conditions as stipulated in Eq. (13). Consequently the cosmological bounce in the higher-derivative theory does not correspond to an analogous bounce in the conformally related Einstein frame.

The above proof is valid only for the cases which involve cosmological bounces in the flat FRW universe. If one takes the spatial curvature of the FRW universe to be non-zero one may have bounces in both the frames only when the spatial curvature is positive, $k=1$. A set of sufficient conditions predicting simultaneous bounces in both the frames are presented in the following discussion. If the bouncing conditions in the Einstein frame are satisfied then there will always be a simultaneous bounce in the Jordan frame when

$$
\varphi_{0}^{\prime}=0, \quad \varphi_{0}^{\prime \prime}<0
$$

at $\tilde{t}=06$. We see from Eq. (40) that if $\varphi_{0}^{\prime}(0)=0$ then both $H$ and $\tilde{H}$ can be zero simultaneously at $t=\tilde{t}=0$. If $\varphi_{0}^{\prime}(0)$ is indeed zero then $\mathrm{Eq}$ (43) reduces to,

$$
\tilde{H}^{\prime}(0)=\frac{k}{\tilde{a}^{2}(0)}-\frac{\kappa}{2}[\tilde{\rho}(0)+\tilde{P}(0)] .
$$

The above equation shows that one of the prerequisites for a bouncing solution in the Einstein frame is $k>0$ such that $\tilde{H}^{\prime}(0)$ can be positive. Differentiating Eq. 40 with respect to the cosmological time, $t$, we get that at $t=\tilde{t}=0$,

$$
\dot{H}=F\left(\tilde{H}^{\prime}-\sqrt{\frac{\kappa}{6}} \varphi_{0}^{\prime \prime}\right),
$$

which implies that a sufficient condition for a simultaneous cosmological bounce in both frames, such that both $\dot{H}$ and $\tilde{H}^{\prime}$ remain positive at $t=\tilde{t}=0$, is $\varphi_{0}^{\prime \prime}(0)<0$.

\footnotetext{
${ }^{6}$ Here we have represented the conditions of a simultaneous bounce in the Einstein frame variables. If one wishes one may easily express these equations in terms of the Jordan frame variable $R$ and its time derivatives by using Eq. $(32)$ and the form of $F(R)$.
} 


\section{Analysis of the bounces in the Jordan frame}

In this section we discuss the cosmological bouncing phenomena in detail using the specific the quadratic gravity $f(R)$ model and verify the claims made so far. To study the bouncing patterns in the flat FRW cosmologies we employ numerical techniques as analytic techniques do not yield simple results for most of the cases in HD theories of gravity. Although, the numerical solutions of the bouncing equations could be done in the Jordan frame itself but we chose to solve the dynamics of the system in the Einstein frame for two important reasons. The first reason is to show specifically the different descriptions, of the same cosmological event, in the two conformally connected frames. This is what we call the relativity of conformally connected frames. The second reason is related to the physical understanding of the bouncing phenomenon in the Jordan frame. Purely a numerical study in the Jordan frame will not answer the typical questions regarding the qualitative nature of the asymmetric bounces. On the other hand it will be seen that the scalar field potential in the Einstein frame and the conditions of cosmological bounce, as translated in the Einstein frame, themselves qualitatively convey the physical nature of the bounces in the conformally connected Jordan frame.

Although we use the Einstein frame to solve our problem, it must be said that the Einstein frame description of the bouncing phenomenon always involves a scalar potential which is unbounded from below. It does not produce a difficult situation in our case because we will see that most of the dynamics of the bouncing system can be effectively described by the behavior of the scalar field near the top of the potential. The HD description of the cosmological bounce does not remain effective once the scalar field has slightly rolled down in the unstable direction.

In the rest of this section we discuss the properties of cosmological bounce in the presence of matter where $f(R)=R+\alpha R^{2}$. Henceforth we will always use $\alpha=-10^{12} M_{P}^{-2}$, for explicit numerical calculations. The reason for choosing this numerical value of $\alpha$ was discussed in the last section. The most probable fluid, which could have been present, during the cosmological bounce can be the perfect radiation fluid. The radiation energy density starts to build up during the contracting phase and peaks during the bounce, after which it starts to get diluted and practically vanishes at the end phase of expansion. In the following analysis we have assumed that bounce occurs in the Jordan frame at $t=0$, which corresponds to $\tilde{t}=0$ in the Einstein frame. In Planck units (where $M_{P}$ will be 


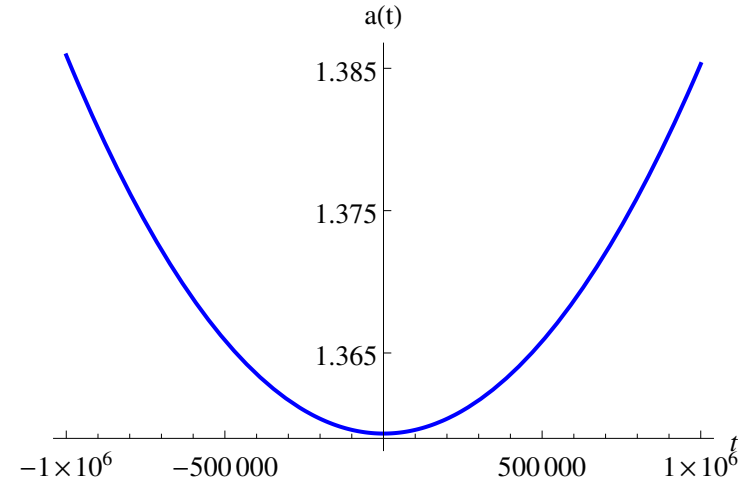

Figure 3: Plot of the Cosmic Scale factor with time in the Jordan frame for quadratic gravity.

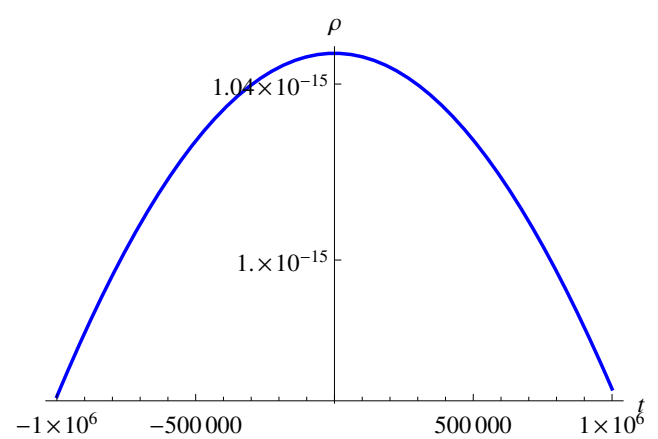

Figure 4: Plot of matter density with time in the Jordan frame for quadratic gravity.

set as one), which we will use henceforth, if one chooses $|\alpha| \sim 10^{12}$ then the time period during which the HD gravity effects become important is given by $-10^{6} \leq t \leq 10^{6} 7$. More over as we recreated the bounce in the Jordan frame from its behavior in the Einstein frame, the relevant conditions of bounce, as the energy density and other variables during bounce, are specified in the Einstein frame. In the Einstein frame one requires only the values of two variables, $\varphi_{0}$ and $\varphi_{0}^{\prime}$, at the bouncing point to predict the cosmological dynamics around the bounce. Translated into the Jordan frame the values of those two variables give us the Ricci scalar $R$ and its time derivative $\dot{R}$ as

$$
R(0)=\frac{e^{\sqrt{2 \kappa / 3} \varphi_{0}(0)}-1}{2 \alpha}, \dot{R}(0)=\frac{\varphi_{0}^{\prime}(0)}{2 \alpha} \sqrt{\frac{2 \kappa}{3}}(1+2 \alpha R(0))^{\frac{3}{2}},
$$

at the bouncing point. In the Jordan frame these two values specify the cosmological state at the bouncing time.

The plot in, Fig. 3, shows the variation of the scale factor during the cosmological bounce whereas Fig. 4 shows the variation of the radiation energy density during the bounce. From these figures it is apparent that the cosmological bounce in this case is slightly asymmetric. The cause of this asymmetry is related to the

\footnotetext{
${ }^{7}$ In conventional units, this time period is roughly $-10^{-37} \mathrm{~s}$ to $10^{-37} \mathrm{~s}$. Although we use the same time interval $-10^{6} \leq t \leq 10^{6}$ in both the Einstein frame and the Jordan frame actually the time-interval in the Einstein frame gets very slightly stretched in the Jordan frame due to the relation between $t$ and $\tilde{t}$. In this article we omit this stretching and work with the same time-interval in both the frames.
} 


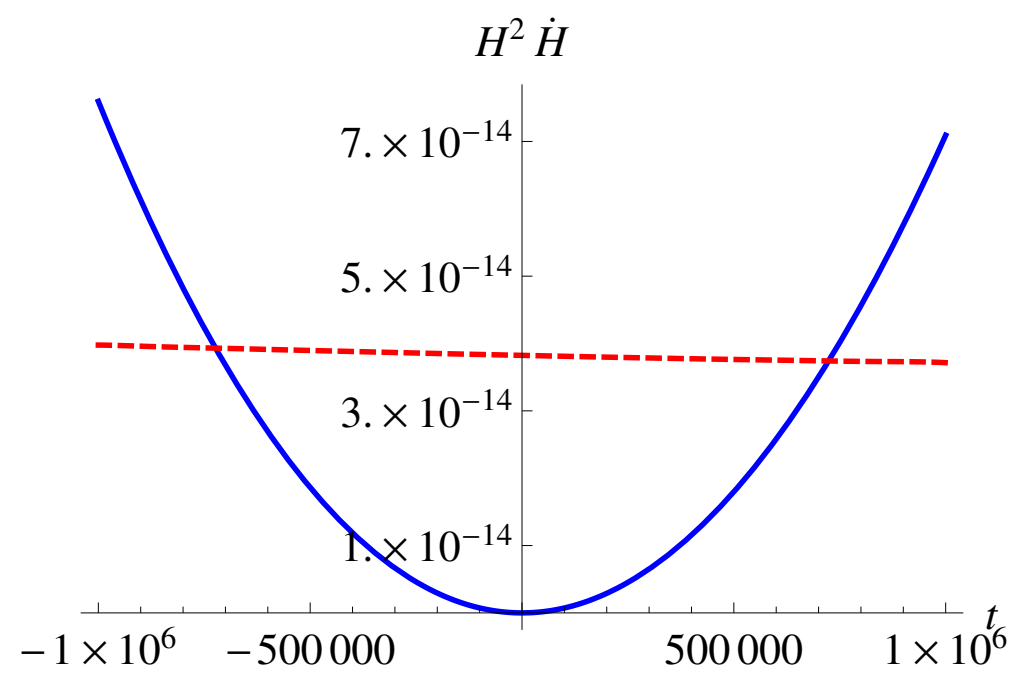

Figure 5: Plot of the square of the Hubble parameter and its time derivative with respect to time in the Jordan frame for quadratic gravity model. The blue solid line represents $H^{2} \times 50$ and the red dashed line represents $\dot{H}$.

values of $\varphi_{0}$ and $\varphi_{0}^{\prime}$ at $\tilde{t}=0$ in the Einstein frame and will be explained in the next section.

Fig. 5 shows, the variation of the square of the Hubble parameter and the time derivative of the Hubble parameter, with respect to time in the Jordan frame during the bouncing period. During the bouncing period it is seen that the time derivative of the Hubble parameter is practically a constant. During the start of the contracting phase of the universe $\dot{H}<H^{2}$ and again during the end of the expansion phase after bounce $\dot{H}<H^{2}$, indicating that the initiation of the radiation dominated bouncing phase may be approximated by a deflationary period and the end of the bouncing phase may be approximated by an inflationary regime.

\section{The corresponding analysis in the Einstein frame}

In this section we specify the method by which we obtained the bouncing solutions. The problem was to solve the system in the Einstein frame and then using the methods in section 3, convert the solutions to the Jordan frame. It was stated before that for the flat universe, one cannot have cosmological bounces in both 


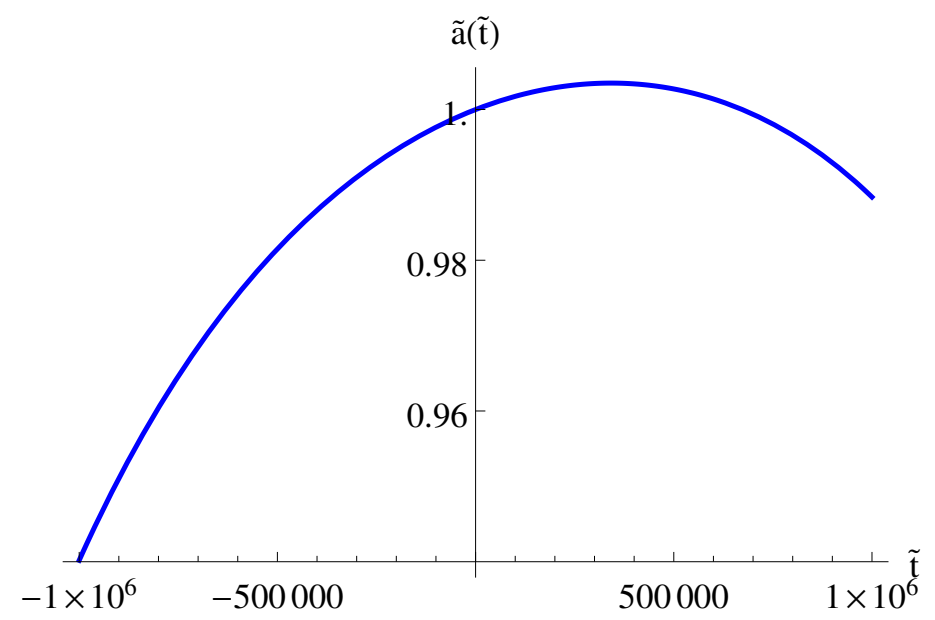

Figure 6: The figure shows the plot of $\tilde{a}(t)$ with $\tilde{t}$ in Einstein frame for the case where the bounce is induced by radiation.

the Jordan and Einstein frames. This statement can be observed by the behavior of the scale factors in the corresponding figures in the Jordan frame and the Einstein frame. The figures, Fig. 3 and Fig. 6 show the differences for the case of quadratic gravity models where bounce occurs in presence of a radiation fluid, in the Jordan frame. In this section we specify the Einstein frame description of the events which correspond to the cosmological bounces in the Jordan frame.

In this section we mainly describe the Einstein frame behavior of quadratic gravity, where $f(R)=R+\alpha R^{2}$. From Fig. 6 it is clear that there is no bounce in the Einstein frame. In the Einstein frame the universe looks like an expanding phase initially. The expansion gives way to a slow contraction during the end of the stipulated time period in which the HD theory is effective. From the plot of the potential of the scalar field $\varphi_{0}$ given in Fig. 2 one can have an idea of the behavior of this case in the Einstein frame. For positive values of $\varphi_{0}$, the Ricci scalar in the Jordan frame turns out to be negative (for a negative $\alpha$ ) ruling out any possibility of a bounce.

The plot of the scalar field potential, Fig. 2, shows that the scalar field must remain sub-Planckian, $\left|\varphi_{0}\right|<M_{P}$, through out the bouncing period. The scalar field potential for negative values of $\varphi_{0}$ is not bounded from below. For a cosmological bounce, in the Jordan frame, the scalar field in the Einstein frame must have some negative value at $\tilde{t}=0$ to start with. If $\varphi_{0}^{\prime}>0$ at $\tilde{t}=0$ then $\varphi_{0}$ increases in time and tries to reach the top of the potential. In this case the 


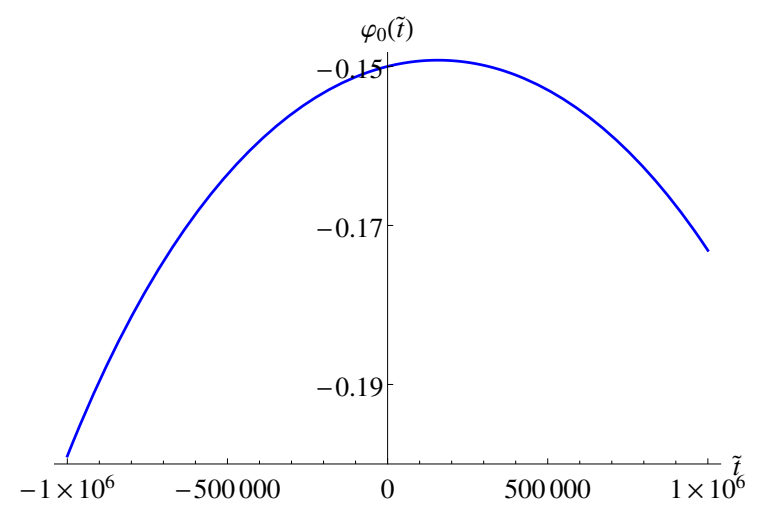

Figure 7: Figure showing the plot of effective scalar field $\varphi_{0}$ with time in Einstein Frame in case of quadratic $f(R)$. In the Einstein frame instead of an initial contraction there is an initial expansion.

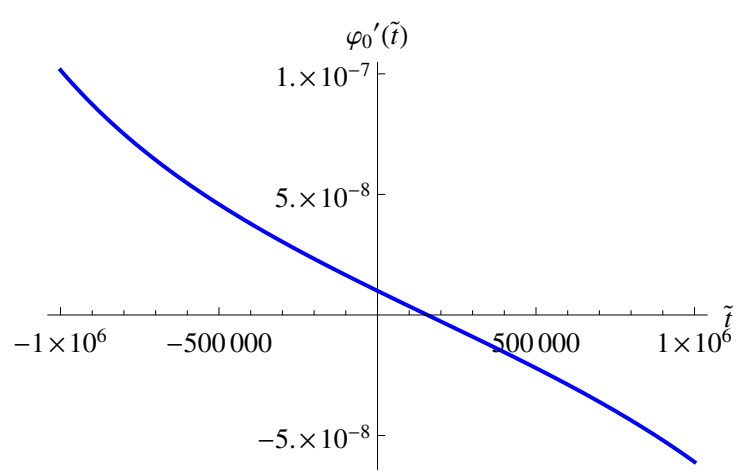

Figure 8: Figure showing the plot of time derivative of effective scalar field $\varphi_{0}$ with time in Einstein Frame in case of quadratic $f(R)$. The figure shows the turning point of $\phi_{0}$ in time where $\phi^{\prime}=0$.

Hubble parameter in the Einstein frame remains positive for a long time as the condition of bounce in the Jordan frame requires $\tilde{H}>0$ at $\tilde{t}=0$ evident from Eq. (51). In its upward journey, the kinetic energy of the scalar field decreases as shown by the decrement of $\varphi_{0}^{\prime}$ in Fig. 8. This slows down the development of the scalar field in its run towards the turning point. There is a point up to which $\varphi_{0}^{\prime}$ remains positive and then it flips sign and the scalar field rolls down the steep side of the potential. The bouncing condition, Eq. (51), and the shape of the potential of the scalar field produces the asymmetric growth of $\varphi_{0}$ in time and this asymmetry is qualitatively reflected in the asymmetric bouncing nature of the scale factor in the Jordan frame. If one wants a perfectly symmetrical bounce in the Jordan frame then one has to implement the condition $\varphi_{0}^{\prime}=0$ at $\tilde{t}=0$ which makes the scalar field's turning point at precisely $\tilde{t}=0$. One may also give a negative value to $\varphi_{0}^{\prime}$ at $\tilde{t}=0$ resulting in an asymmetric bounce where the evolution of the scale factor, in the Jordan frame, slows down in the contracting phase of the universe unlike the one shown in Fig. 3, where $\varphi_{0}^{\prime}>0$ at $\tilde{t}=0$.

For the plots presented in this article, showing radiation dominated bounce in the Jordan frame, we have chosen that at $\tilde{t}=0$

$$
\varphi_{0}=-.15, \quad \varphi_{0}^{\prime}=10^{-8} .
$$

The smallness of $\varphi_{0}^{\prime}$ and the position of $\varphi_{0}$ in the scalar field potential dictates the time scale of the bouncing phenomenon. These two are the only independent 


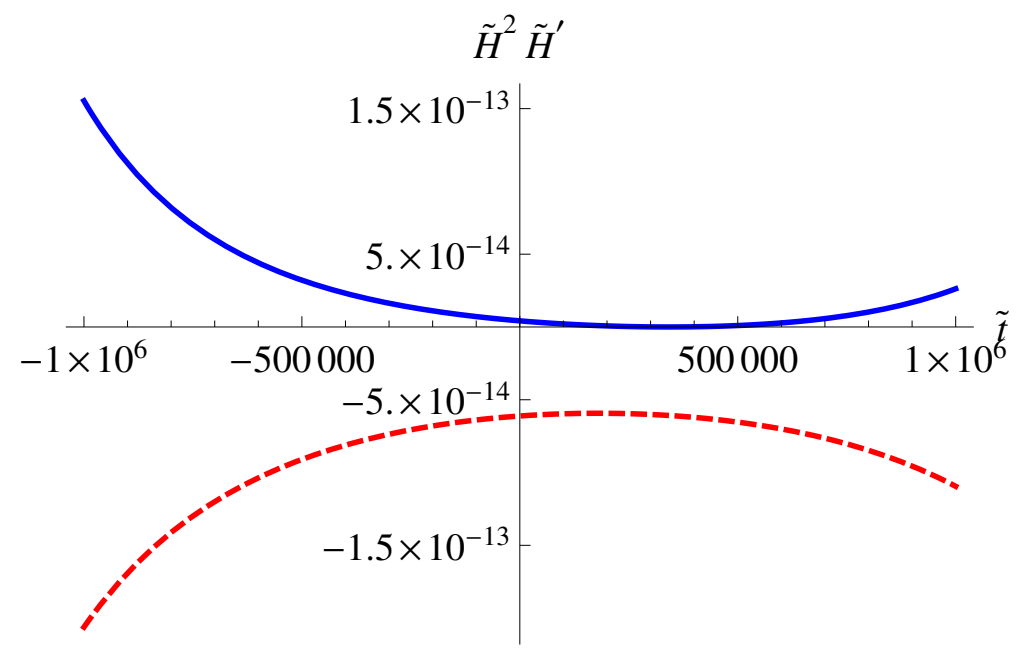

Figure 9: The plot showing how $\tilde{H}^{2}$ and $\tilde{H}^{\prime}$ vary with $\tilde{t}$ in the Einstein frame in case of quadratic $f(R)$. The blue solid line is $\tilde{H}^{2} \times 10$ and the red dashed line is $\tilde{H}^{\prime}$.

quantities, specifying whose values at the time of bounce, dictates the dynamics of the cosmological system uniquely.

The effective ambient hydrodynamic matter in the Einstein frame is modelled by a fluid whose $\omega=1 / 3$, like conventional radiation, but the energy density and pressure of this fluid depends upon the scalar field strength. This coupling of the scalar field to the original hydrodynamic fluid is referred as a non-minimal coupling of the scalar field to matter. The state of hydrodynamic matter at $\tilde{t}=0$ is obtained from the bouncing condition in the Jordan frame $H(t=0)=0$, which implies

$$
\tilde{H}=\sqrt{\frac{\kappa}{6}} \varphi_{0}^{\prime},
$$

at $\tilde{t}=0$ in the Einstein frame, and Eq. 41). The aforementioned two conditions specifies

$$
\tilde{\rho}=-V\left(\varphi_{0}\right)
$$

at $\tilde{t}=0$, giving the energy content in the non-minimally coupled sector. Finally, the plot of the Hubble parameter and its time derivative in the Einstein frame is shown in Fig. 9. 


\section{Scalar metric perturbations in the Einstein frame}

The background bouncing FRW solutions, in the Jordan frame, can have cosmological perturbations and these perturbations evolve in spacetime. Cosmological perturbations in $f(R)$ gravity has been studied in various forms in the Jordan frame and the Einstein frame while dealing with inflationary cosmology or structure formation [31, 41, 44]. In this section we will present the Einstein frame description of the perturbations produced during a cosmological bounce in the Jordan frame where the dynamics of the perturbations is guided by a HD theory of gravity. As like the background bouncing solution these perturbation can also be analyzed in the Einstein frame. Once the solution of the metric perturbations, corresponding to the bouncing solutions in the Jordan frame, are solved in the Einstein frame one may transform the results back to the Jordan frame.

Focussing only on the scalar perturbations one can write the perturbed line element as,

$$
d s^{2}=a^{2}(\eta)\left[-(1+2 \phi) d \eta^{2}+2 B_{, i} d \eta d x^{i}+\left\{(1-2 \psi) \delta_{i j}+2 E_{, i j}\right\} d x^{i} d x^{j}\right],
$$

where $\phi, \psi, B, E$ characterize the scalar perturbations and commas followed by $i$ and $j$ appearing after the functions $B$ and $E$ refer to partial differentiation of the functions with respect to $x^{i}$ or $x^{j}$. One can construct the gauge invariant Bardeen potentials by suitable combinations of the scalar functions as

$$
\Phi=\phi+\frac{1}{a}\left[a\left(B-E^{\prime}\right)\right]^{\prime}, \quad \Psi=\psi-\frac{a^{\prime}}{a}\left(B-E^{\prime}\right) .
$$

It has to be emphasized at this point that the primes over the quantities in this section will mean a derivative with respect to the conformal time. The conformal time remains the same both in the Jordan and the Einstein frames. In terms of gauge invariant Bardeen potentials, the perturbed metric in the Jordan frame is given as

$$
d s^{2}=a^{2}(\eta)\left[-(1+2 \Phi) d \eta^{2}+(1-2 \Psi) \delta_{i j} d x^{i} d x^{j}\right],
$$

where $\Phi \neq \Psi$ in HD theories of gravity. The perturbed metric in the Einstein frame can also be written as

$$
d \tilde{s}^{2}=\tilde{a}^{2}(\eta)\left[-(1+2 \tilde{\Phi}) d \eta^{2}+(1-2 \tilde{\Psi}) \delta_{i j} d x^{i} d x^{j}\right],
$$


As the energy momentum tensor, $\tilde{S}_{\nu}^{\mu}$, as given in Eq. (31) in the Einstein frame is diagonal one has $\tilde{\Phi}=\tilde{\Psi}$. The Bardeen potentials in the Jordan frame can be calculated from $\tilde{\Phi}$ in the following way [43] :

$$
\Phi=-\frac{2}{3}\left(\frac{F^{2}}{F^{\prime} a}\right)\left[\left(\frac{a}{F}\right) \tilde{\Phi}\right]^{\prime}, \quad \Psi=\frac{2}{3}\left(\frac{1}{F F^{\prime} a}\right)\left(a F^{2} \tilde{\Phi}\right)^{\prime} .
$$

Assuming the perturbation in the matter sector to be adiabatic in the Jordan frame, one can write

$$
\delta p^{(g i)}-c_{s}^{2} \delta \rho^{(g i)}=0
$$

For any quantity $q, \delta q^{(g i)} \equiv \delta q+q_{0}^{\prime}\left(B-E^{\prime}\right)$, where $q_{0}$ denotes the unperturbed quantity. Replacing $\rho, p$ by $\tilde{\rho}, \tilde{p}$ in the Eq. (58), and keeping in mind that $\delta F / F=$ $\sqrt{2 \kappa / 3} \delta \varphi$, where $\varphi(\eta, \mathbf{x})=\varphi_{0}(\eta)+\delta \varphi(\eta, \mathbf{x})$ one obtains

$$
\delta \tilde{p}^{(g i)}-c_{s}^{2} \delta \tilde{\rho}^{(g i)}=2 \sqrt{\frac{2 \kappa}{3}} \tilde{\rho}_{0}\left(c_{s}^{2}-\omega\right) \delta \varphi^{(g i)},
$$

where $\tilde{\rho}_{0}$ is the background energy density of the effective fluid. Till the last section this parameter was not represented with the zero subscript but in this section we use this convention to differentiate the background values from their perturbations. The above equation shows that if one has a single component barotropic fluid for which $c_{s}^{2}=\omega$ in the Jordan frame, the hydrodynamic perturbations produced in the Einstein frame are also adiabatic. In terms of the Hubble parameter $\tilde{\mathcal{H}}$, now represented as a function of the conformal time, the gauge invariant perturbed field equations in the Einstein frame are:

$$
\begin{aligned}
-3 \tilde{\mathcal{H}}\left(\tilde{\mathcal{H}} \tilde{\Phi}+\tilde{\Phi}^{\prime}\right)+\nabla^{2} \tilde{\Phi}= & \frac{\kappa}{2}\left(\tilde{a}^{2} \delta \tilde{\rho}^{(g i)}-\varphi_{0}^{\prime 2} \tilde{\Phi}+\varphi_{0}^{\prime} \delta \varphi^{(g i)^{\prime}}\right. \\
& \left.+\tilde{a}^{2} V_{, \varphi_{0}} \delta \varphi^{(g i)}\right) \\
\left(2 \tilde{\mathcal{H}}^{\prime}+\tilde{\mathcal{H}}^{2}\right) \tilde{\Phi}+\tilde{\Phi}^{\prime \prime}+3 \tilde{\mathcal{H}} \tilde{\Phi}^{\prime}= & \frac{\kappa}{2}\left(\tilde{a}^{2} \delta \tilde{p}^{(g i)}-\tilde{\Phi} \varphi_{0}^{\prime 2}+\varphi_{0}^{\prime} \delta \varphi^{(g i)^{\prime}}\right. \\
& \left.-\tilde{a}^{2} V_{, \varphi_{0}} \delta \varphi^{(g i)}\right),
\end{aligned}
$$

where in the above equations $V_{, \varphi_{0}}$ specifies the derivative of the scalar field potential with respect to $\varphi_{0}$. Multiplying Eq. (60) by $c_{s}^{2}$ and subtracting the resultant from Eq. (61) yields:

$$
\begin{aligned}
& \tilde{\Phi}^{\prime \prime}-c_{s}^{2} \nabla^{2} \tilde{\Phi}+\left(2 \tilde{\mathcal{H}}^{\prime}+\tilde{\mathcal{H}}^{2}\right) \tilde{\Phi}+3 \tilde{\mathcal{H}} \tilde{\Phi}^{\prime}+3 c_{s}^{2} \tilde{\mathcal{H}}\left(\tilde{\mathcal{H}} \tilde{\Phi}+\tilde{\Phi}^{\prime}\right)=-\frac{\kappa}{2} \tilde{\Phi} \varphi_{0}^{\prime 2}\left(1-c_{s}^{2}\right) \\
& +\frac{\kappa}{2} \varphi_{0}^{\prime}\left(1-c_{s}^{2}\right) \delta \varphi^{(g i)^{\prime}}-\frac{\kappa \tilde{a}^{2}}{2} V_{, \varphi_{0}}\left(1+c_{s}^{2}\right) \delta \varphi^{(g i)}
\end{aligned}
$$


In the rest frame of the hydrodynamic fluid one can write

$$
\frac{\kappa}{2} \delta \varphi^{(g i)}=\frac{1}{\varphi_{0}^{\prime}}\left[\tilde{\Phi}^{\prime}+\tilde{\mathcal{H}} \tilde{\Phi}\right]
$$

and taking it's derivative with respect to $\eta$ one gets

$$
\frac{\kappa}{2} \varphi_{0}^{\prime} \delta \varphi^{(g i)^{\prime}}=\left(\tilde{\mathcal{H}}^{\prime}-\frac{\varphi_{0}^{\prime \prime}}{\varphi_{0}^{\prime}} \tilde{\mathcal{H}}\right) \tilde{\Phi}+\left(\tilde{\mathcal{H}}-\frac{\varphi_{0}^{\prime \prime}}{\varphi_{0}^{\prime}}\right) \tilde{\Phi}^{\prime}+\tilde{\Phi}^{\prime \prime} .
$$

Using the above equation, the form of Eq. (44) written in conformal time and

$$
\frac{\kappa}{2} \varphi_{0}^{\prime 2}=\tilde{\mathcal{H}}^{2}-\tilde{\mathcal{H}}^{\prime}-\frac{\kappa \tilde{a}^{2}}{2}\left(\tilde{\rho}_{0}+\tilde{p_{0}}\right),
$$

which is obtained from the Einstein equations for the background spacetime, in Eq. 62 we obtain the equation for scalar perturbation in the Einstein frame as:

$$
\begin{gathered}
c_{s}^{2}\left(\tilde{\Phi}^{\prime \prime}-\nabla^{2} \tilde{\Phi}\right)+\left[2 c_{s}^{2}\left(\tilde{\mathcal{H}}-\frac{\varphi_{0}^{\prime \prime}}{\varphi_{0}^{\prime}}\right)+\frac{\tilde{a}^{2}}{\varphi_{0}^{\prime}} \sqrt{\frac{\kappa}{6}} \tilde{\rho}_{0}\left(1+c_{s}^{2}\right)\left(1-3 c_{s}^{2}\right)\right] \tilde{\Phi}^{\prime} \\
+\left[2\left(\tilde{\mathcal{H}}^{\prime}-\tilde{\mathcal{H}} \frac{\varphi_{0}^{\prime \prime}}{\varphi_{0}^{\prime}}\right) c_{s}^{2}+\tilde{a}^{2} \tilde{\rho}_{0}\left(1+c_{s}^{2}\right)\left\{\sqrt{\frac{\kappa}{6}} \frac{\tilde{\mathcal{H}}}{\varphi_{0}^{\prime}}\left(1-3 c_{s}^{2}\right)-\frac{\kappa}{2}\left(1-c_{s}^{2}\right)\right\}\right] \tilde{\Phi}=0,
\end{gathered}
$$

where $\tilde{\rho}_{0}$ and $\tilde{p}_{0}$ are the background energy density and pressure of the effective fluid. For a single barotropic fluid in the Jordan frame $\tilde{p}_{0}=c_{s}^{2} \tilde{\rho}_{0}$ where $c_{s}^{2}$ is the sound velocity in the Jordan frame. In absence of any hydrodynamic fluid in the Jordan frame the above equation becomes

$$
\tilde{\Phi}^{\prime \prime}-\nabla^{2} \tilde{\Phi}+2\left(\tilde{\mathcal{H}}-\frac{\varphi_{0}^{\prime \prime}}{\varphi_{0}^{\prime}}\right) \tilde{\Phi}^{\prime}+\left[2\left(\tilde{\mathcal{H}}^{\prime}-\tilde{\mathcal{H}} \frac{\varphi_{0}^{\prime \prime}}{\varphi_{0}^{\prime}}\right)\right] \tilde{\Phi}=0,
$$

which is equivalent to the corresponding equation for scalar metric perturbation in Ref. [35] where the authors used the background FRW solution to have a positive curvature. If the curvature term in Ref. [35] is dropped then the equation for the scalar metric perturbation equation matches exactly with the above equation 8 ,

\footnotetext{
${ }^{8}$ Actually Eq. 66 is written for the case where the background FRW solution is spatially flat, $k=0$. In the general case, where $k \neq 0$ the coefficient function multiplying $\tilde{\Phi}$ in Eq. 66. will have an extra piece, $-4 k$, added. From this modified form of Eq. 66 it can be easily verified that it reduces to the corresponding equation for the metric perturbation in Ref. [35]. In Ref. 35 as the authors used positively curved spatial hypersurfaces in FRW spacetime there they used $\nabla^{2} \tilde{\Phi}=-n(n+2) \tilde{\Phi}$ where $n$ is an integer.
} 
If the background hydrodynamic fluid is made up of radiation then Eq. 65 takes a simpler form as

$$
\tilde{\Phi}^{\prime \prime}-\nabla^{2} \tilde{\Phi}+2\left(\tilde{\mathcal{H}}-\frac{\varphi_{0}^{\prime \prime}}{\varphi_{0}^{\prime}}\right) \tilde{\Phi}^{\prime}+\left[2\left(\tilde{\mathcal{H}}^{\prime}-\tilde{\mathcal{H}} \frac{\varphi_{0}^{\prime \prime}}{\varphi_{0}^{\prime}}\right)-\frac{4 \kappa}{3} \tilde{a}^{2} \tilde{\rho}_{0}\right] \tilde{\Phi}=0
$$

where we have used $c_{s}^{2}=1 / 3$. One can also use the background fluid to be dust like and see the evolution of the perturbations across the bounce. Cosmological perturbations in presence of hydrodynamic matter in general predicts that for a dust filled universe the perturbation equations become a second order differential equation in time which is independent of the wave number of the perturbations. This happens, independently of the underlying theory of gravity, because the wave number dependent terms in the perturbation equation come with a factor of $c_{s}^{2}$ multiplied to it. In the present case, one may see that the above argument is slightly modified. The form of Eq. (65) predicts that in a dust filled universe the differential equation predicting the evolution of the scalar perturbation becomes a first order differential equation, in time, which is independent of the wave number of the perturbation. From a quantum mechanical point of view this poses a problem because in those cases it is difficult to impose initial conditions on the perturbations and so the general ansatz is to hold $c_{s}^{2} \rightarrow 0$, not exactly zero, such that some wave number dependence persists in the theory. In the next subsection we will present the numerical evolution of the, classical perturbations, in the Jordan frame for which the sound velocity will be taken arbitrarily small but not exactly zero. The issue of quantization of the perturbations will be dealt in a future publication and we will not discuss about it in this article.

Before leaving the topic of cosmological perturbations in the Einstein frame it is important to note one interesting property about the main evolution equation Eq. (65). The general technique to solve differential equations, like Eq. (65), is to expand $\tilde{\Phi}(\eta, \mathbf{x})$ in the eigenfunction basis of operator $\nabla^{2}$. In such cases the Laplacian operator is replaced by the eigenvalue of the operator. Ultimately one can transform Eq. (65) into a second order differential equation for $\tilde{\Phi}(\eta)$ in which the coefficients of $\tilde{\Phi}^{\prime}$ and $\tilde{\Phi}$ will be functions of the form $\varphi_{0}^{\prime \prime} / \varphi_{0}^{\prime}, 1 / \varphi_{0}^{\prime}$ or $\mathcal{H} \varphi_{0}^{\prime \prime} / \varphi_{0}^{\prime}$. In our analysis of the behavior of the scalar field in the Einstein frame, in section 5, it was pointed out that during the bouncing process $\varphi_{0}^{\prime}$ always reaches zero at some time and that is the turning point for $\varphi_{0}$. From the form of Eq. 65 it seems that coefficients in the differential equation becomes singular at the value of $\eta$ when $\varphi_{0}^{\prime}=0$. But under closer inspection it becomes clear 
that these singular points are actually regular singular points of the equation. In general if $\varphi_{0}^{\prime}\left(\eta_{0}\right)=0$ it can be easily seen that $\lim _{\eta \rightarrow \eta_{0}}\left(\left(\eta-\eta_{0}\right) g(\eta) / \varphi_{0}^{\prime}\right)$, or $\lim _{\eta \rightarrow \eta_{0}}\left(\left(\eta-\eta_{0}\right)^{2} g(\eta) / \varphi_{0}^{\prime}\right)$ where $g(\eta)$ is some function of $\eta$, is always nonsingular if $\varphi_{0}^{\prime \prime}\left(\eta_{0}\right) \neq 0$. One must always have $\varphi_{0}^{\prime \prime}\left(\eta_{0}\right) \neq 0$ as $\eta_{0}$ is a turning point of $\varphi_{0}$ where the rate of change of of $\varphi_{0}^{\prime}$ is non zero. These fact implies that there must exist well behaved solutions of the cosmological perturbation evolution equation throughout the bouncing regime.

\subsection{Numerical evolution of the scalar metric perturba- tions across the bounce}

In this subsection we present the numerical evolution of the growth of the scalar cosmological perturbations in radiation dominated and matter dominated universes whose cosmology is governed by quadratic $f(R)$ gravity during a brief bouncing period centered at $\eta=0$. The results yield plots of $\Phi$ and $\Psi$ in the Jordan frame for specific values of the wave number $k$ of the perturbations. To calculate the perturbations in the Jordan frame one has to use the relations, as given in Eq. (57), which connect $\tilde{\Phi}$ in the Einstein frame to the corresponding perturbation potentials in the Jordan frame. The relations in Eq. (57) reveals that the Jordan frame perturbation potentials can diverge when $F^{\prime}=0$. It is known that $F^{\prime}$ must vanish at one instant during the bouncing period. Mathematically one cannot use the Einstein frame description of the perturbations to track the development of the metric perturbations in the Jordan frame completely. For an asymmetric bounce, when $F^{\prime}=0$ at an instant, the Jordan frame potentials will become singular when they are calculated using Eq. (57). The singularity of the Jordan frame metric perturbations at a particular instant of time is purely an artefact of the transformation relations of the perturbations in different conformal frames as given in Eq. (57) and does not represent a real singularity of the perturbations as no such singularity in the evolution of the metric perturbation $\tilde{\Phi}$ appears in the Einstein frame. The singularities in the relations in Eq. (57) do not arise for the symmetric bounce cases when the perturbation modes are also symmetric in time in the Einstein frame. In this case $F^{\prime}=a^{\prime}=\tilde{\Phi}^{\prime}=0$ at $\eta=0$ and one can easily verify that the connecting relations in the perturbations remain well behaved throughout the bouncing phase. For an asymmetric perturbation mode in the case of a symmetric background evolution one can have

$\tilde{\Phi}^{\prime} \neq 0$ at $\eta=0$ and in such a case the discontinuity of the Jordan frame per- 


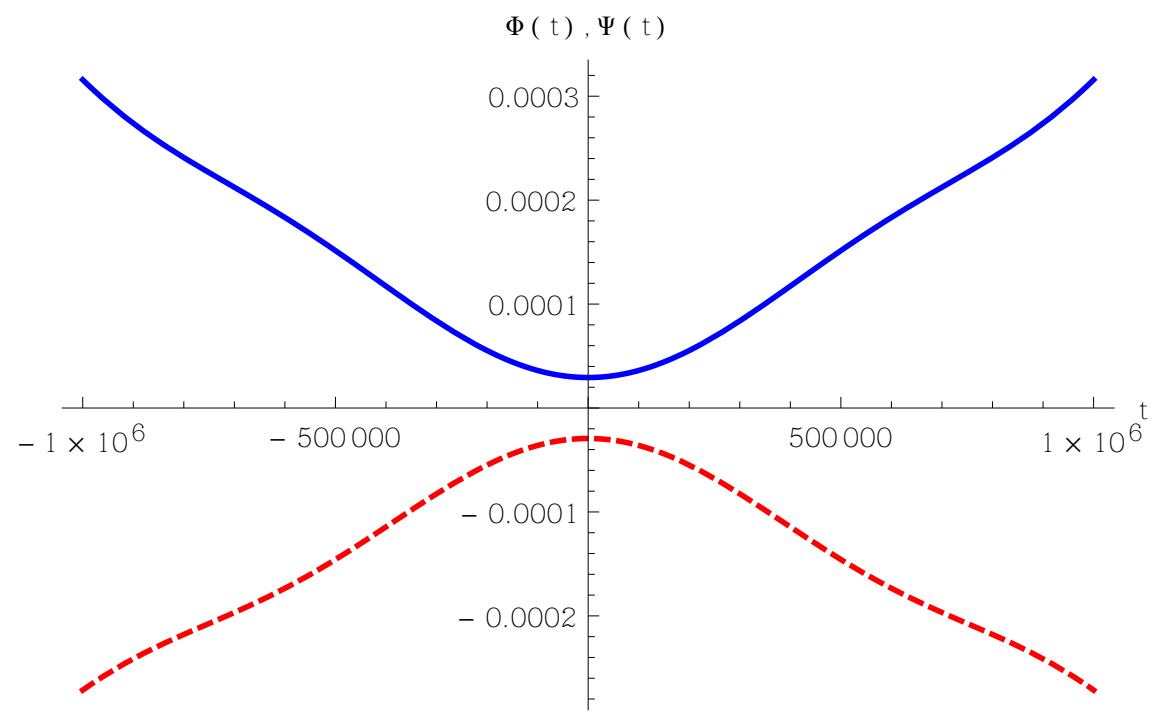

Figure 10: The plot showing the evolution of $\Phi$ and $\Psi$ in the Jordan frame for radiation induced cosmological bounce. The blue coloured solid curve represents $\Phi$ and the red dashed curve represents $\Psi$. The perturbations are plotted with respect to normal Jordan frame time variable $t$.

turbation potentials can reappear. The above discussion shows that the Einstein frame description of the evolution of the perturbations can be used to study the perturbations in the HD theory as the divergence of the scalar functions in the Jordan frame (if present) are purely local (about an instant). In this subsection we will be presenting the perturbation evolutions for the symmetric bounces as well as the asymmetric bounces for various kinds of hydrodynamic fluid in the Jordan frame.

At first we study the symmetric bounce case where the perturbation modes are also symmetric in time. In this case it is better to specify the initial conditions for the numerical evolution of the perturbations at $\eta=0$. In Fig. 10 we show the evolution of the scalar metric perturbations in the Jordan frame where the bounce is triggered by a radiation fluid. The wave number for the perturbation mode, $k=10^{-12}$, correspond to a superhorizon mode at $t=-10^{6}$. Although the perturbation calculations were done using conformal time we represent the final evolutions of the perturbations in the Jordan frame using the Jordan frame time variable $t$. To generate the numerical solutions we have assumed that the bounce is symmetric, where for the background evolution $\phi(0)=-0.15, \phi^{\prime}(0)=0$ and for the perturbations $\tilde{\Phi}(0)=10^{-8}$ and $\tilde{\Phi}^{\prime}(0)=0$ in the Einstein frame. The 


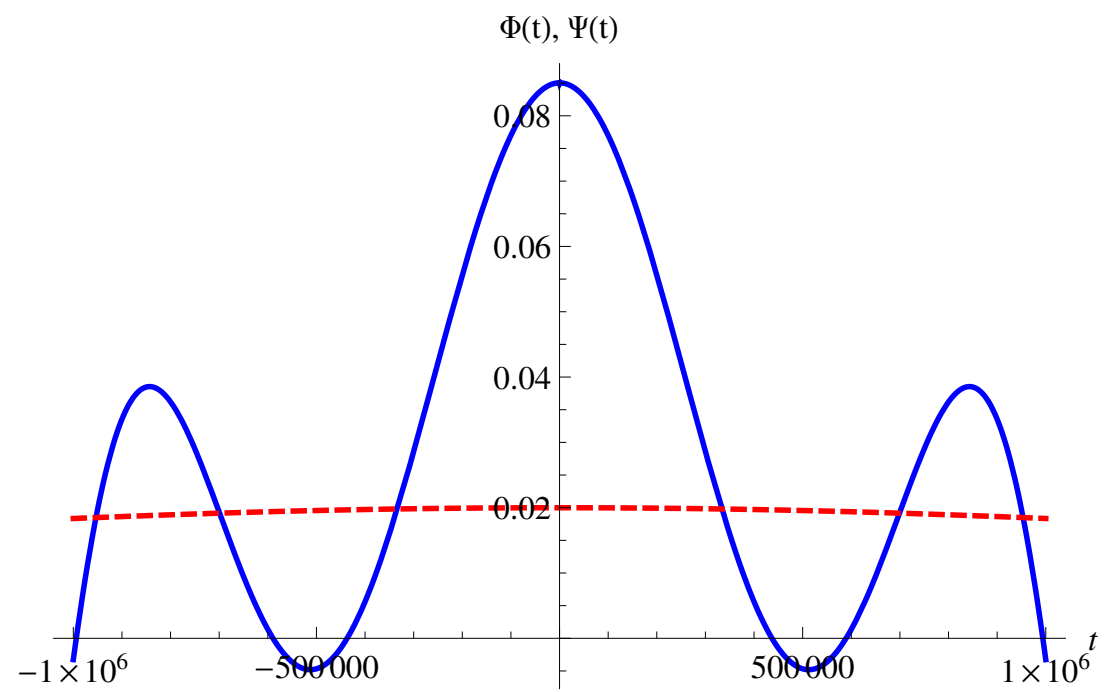

Figure 11: The plot showing the evolution of $\Phi$ and $\Psi$ in the Jordan frame for dust matter induced bounce. The blue coloured solid curve represents $10^{4} \times \Phi$, and the red dashed curve represents $\Psi$.

magnitude of $\tilde{\Phi}(0)$ in the Einstein frame is chosen in such a fashion that the magnitude of the metric perturbations in the Jordan frame are of the order of $10^{-4}$. From Fig. 10 it is seen that the quantities $\Phi$ and $\Psi$ practically remains constant during the bouncing period, although a slight variation is noted in their magnitudes. The evolution of the perturbations also remain symmetric in time.

The corresponding perturbation evolution in the dust dominated bouncing universe is shown in Fig. 11. In this case also $k=10^{-12}$, for the perturbation mode, which corresponds to a superhorizon mode in the beginning phase of the bouncing period. To keep the $k$ dependent terms in the perturbation evolution we have used $c_{s}^{2}=10^{-8}$ in Eq. (65). In this case also it is noticed that the symmetry of the bounce is reflected in the perturbation modes. Although $\Phi$ oscillates in the bouncing period but its overall variation in the that period is negligible. The perturbations remain practically constant through the bouncing phase. In this case initial values used to determine the perturbations in the Einstein frame are, $\tilde{\Phi}(0)=10^{-2}$ and $\tilde{\Phi}^{\prime}(0)=0$ and the background values of $\phi(0)$ and $\phi^{\prime}(0)$ remain the same as in the case of radiation induced bounce.

Next we present the more general results for both symmetric and asymmetric bounces where the perturbations may not be symmetric. In this cases we set the initial conditions for the perturbation at $\tilde{t}=-10^{6}$ where time is expressed in 


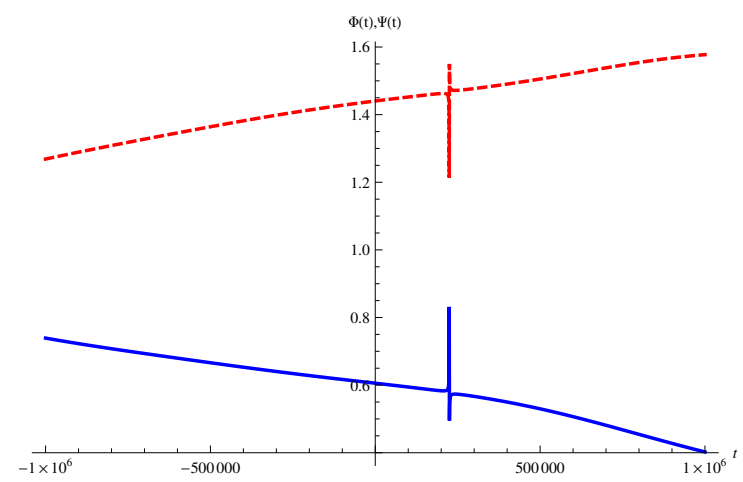

Figure 12: Metric perturbation potentials, $\Phi$ (solid blue) and $\Psi$ (dashed red), in the Jordan frame for an asymmetric radiation induced bounce where $\tilde{\Phi}=1$, and $\tilde{\Phi}^{\prime}=0$ at $\tilde{t}=-10^{6}$.

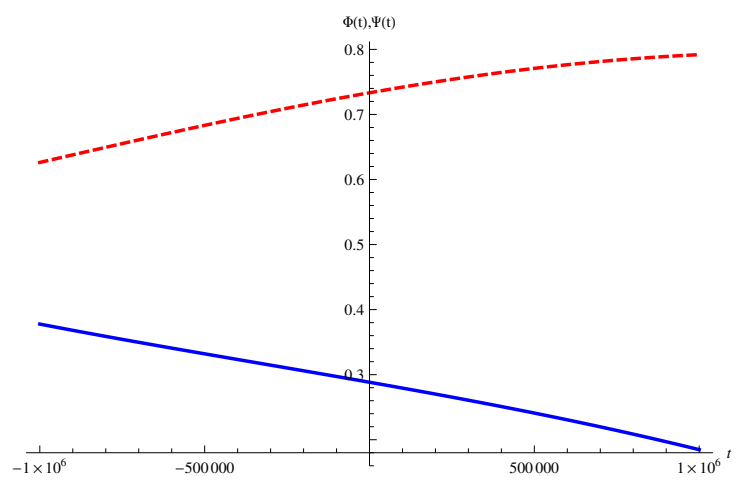

Figure 13: Metric perturbation potentials, $\Phi$ (solid blue) and $\Psi$ (dashed red), in the Jordan frame for symmetric radiation induced bounce where $\tilde{\Phi}=0.5$, and $\tilde{\Phi}^{\prime}=0$ at $\tilde{t}=-10^{6}$.

Planck units. In Figs. 12 and 13 we present the perturbation evolutions for an asymmetric and symmetric radiation induced bounce in quadratic gravity. Here we immediately see that in Fig. 12 there is a sharp jump of the metric perturbations near the bouncing point and one can verify that at that time $F^{\prime}(R)=0$. The jumps are shown to be finite in the plots because of limitation of the numerical approach due to which the exact value of $F^{\prime}(R)=0$ is never reached. But Fig. 12 shows that except one single point the evolution of the perturbations in the Jordan frame is perfectly well defined for an asymmetric bounce. The background evolution for the asymmetric bounce cases discussed here and henceforth will use the initial conditions as specified in Eq. 50 and the symmetric bounce initial conditions remain the same as discussed before in this subsection. The wave numbers for the modes will we chosen to be $k=10^{-12}$ throughout. The perturbations are seen to be nearly constant in the Jordan frame. Interestingly we do not observe the discontinuities of $\Phi$ and $\Psi$ at $t=0$ in the symmetric bounce. They should in principle be present as now the initial conditions for the perturbation evolutions do not ensure that $\tilde{\Phi}^{\prime}=0$ at $\eta=0$. They remain unobservable because the amplitude of the discontinuous jump at $t=0$ for both the perturbation potentials turns out to be much smaller than the original functional values of these variables at $t=0$ and consequently they remain hidden and the perturbation potentials look smooth at $t=0$ in the Jordan frame. We will show that in the matter induced bounce background one will be able to observe these 


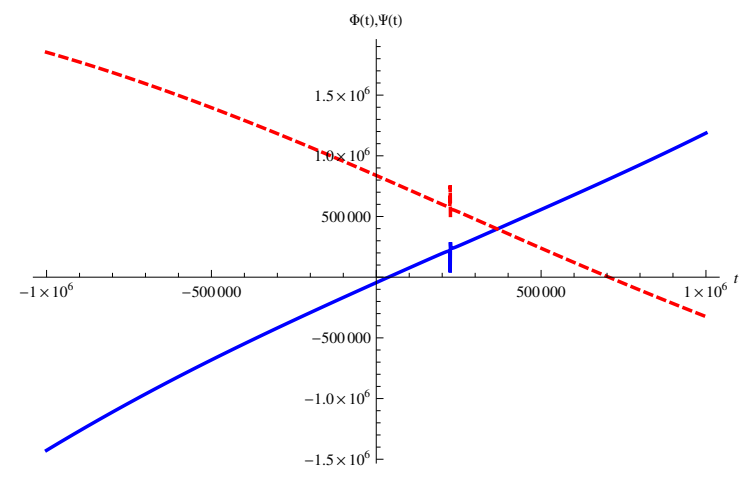

Figure 14: Metric perturbation potentials, $\Phi$ (solid blue) and $\Psi$ (dashed red), in the Jordan frame for an asymmetric radiation induced bounce where $\tilde{\Phi}=0$, and $\tilde{\Phi}^{\prime}=1$ at $\tilde{t}=-10^{6}$.

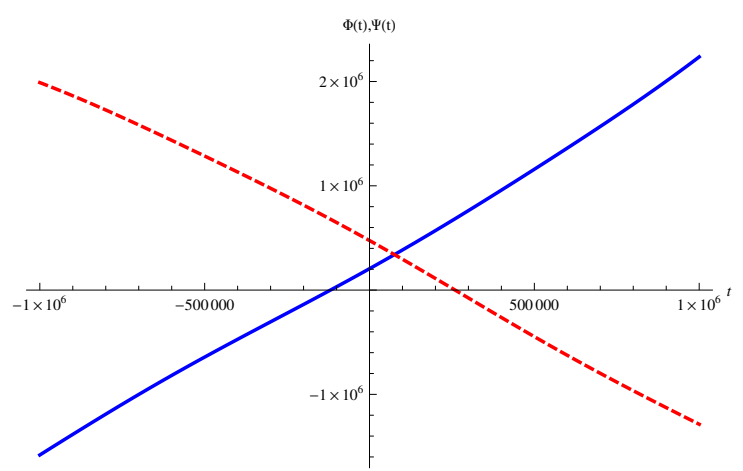

Figure 15: Metric perturbation potentials, $\Phi$ (solid blue) and $\Psi$ (dashed red), in the Jordan frame for symmetric radiation induced bounce where $\tilde{\Phi}=0$, and $\tilde{\Phi}^{\prime}=1$ at $\tilde{t}=-10^{6}$.

discontinuous jumps in $\Phi$ even for a symmetric bounce background as there the amplitude of the perturbing potential $\Phi$ is itself very small and the amplitude of the "apparent" finite discontinuity does not remain hidden.

All the initial conditions set in the Einstein frame do not translate into well behaved constant perturbations in the Jordan frame. Our numerical results show that the results are particularly sensitive to the initial value of $\tilde{\Phi}^{\prime}$. For large values of $\tilde{\Phi}^{\prime}$ as initial condition one gets the perturbing potentials $\Phi$ and $\Psi$ to be very large and the theory does not remain perturbative. This fact is shown in the specimen plots in Figs. 14 and 15 where the values of the perturbing potentials are practically diverging throughout the bouncing regime. The result is not surprising as if one sets $\tilde{\Phi}^{\prime}=1$ at the initial stage then the perturbation potential $\tilde{\Phi}$ in the Einstein frame is steeply increasing with time which may attain a very high value very quickly. The transformed Jordan frame perturbation potentials reflect the unboundedness of the perturbation in the Einstein frame. The plots in Fig. 14 and Figs. 15 and the discussion above shows that the interesting initial conditions, in the Einstein frame, which give rise to physically reliable scalar metric perturbation evolution in the Jordan frame must reject high values of $\tilde{\Phi}^{\prime}$. On the other hand our numerical evolutions show that the results are also dependent on the magnitude of $\tilde{\Phi}$ initially. If $\tilde{\Phi} \sim 1$ then the perturbation potentials are approximately near to one, as in Fig. 12, but may grow as time increases in some other cases. In the perturbative regime of the potential, $\tilde{\Phi} \lesssim 1$, 


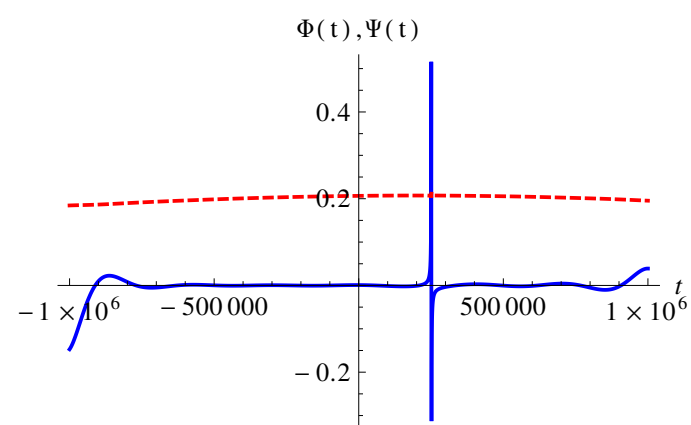

Figure 16: Metric perturbation potentials, $\Phi \times 10^{2}$ (solid blue) and $\Psi$ (dashed red), in the Jordan frame for an asymmetric dust matter induced bounce where $\tilde{\Phi}=0$, and $\tilde{\Phi}^{\prime}=1$ at $\tilde{t}=-10^{6}$.

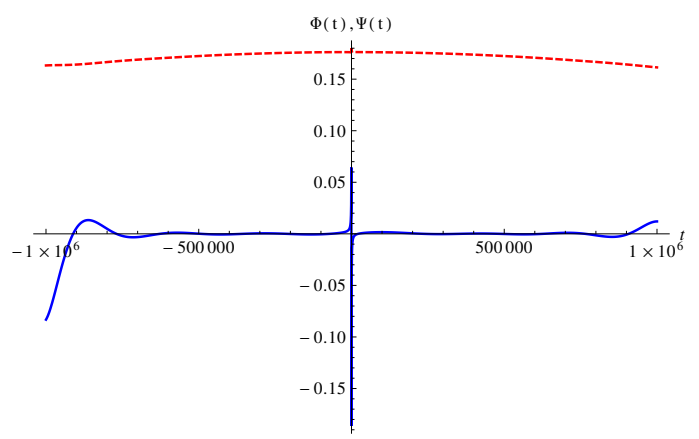

Figure 17: Metric perturbation potentials, $\Phi \times 50$ (solid blue) and $\Psi$ (dashed red), in the Jordan frame for symmetric dust matter induced bounce where $\tilde{\Phi}=0$, and $\tilde{\Phi}^{\prime}=1$ at $\tilde{t}=-10^{6}$.

and its derivative $\tilde{\Phi}^{\prime} \lesssim 1$ the growth of $\Phi$ and $\Psi$ in the Jordan frame remain well behaved.

The pattern of time evolution of the perturbation potentials for the dust induced bounce is similar to the radiation induced bounce except a couple of points. We had commented earlier that for a matter induced bounce the perturbation evolution equation in the Einstein frame becomes more like a first order differential equation in $\tilde{\Phi}$. For the sake of the quantum mechanical initial conditions it was assumed that for dust matter perturbations it is safe to take $c_{s}^{2} \rightarrow 0$ (where $c_{s}^{2} \neq 0$ ) and consequently in our plots we have always assumed $c_{s}^{2}=10^{-8}$. In such cases it can be show that the effect of the second derivative of $\tilde{\Phi}$ with respect to the conformal time in Eq. (65) becomes negligible and consequently the perturbation evolution equation behaves more like a first order differential equation in $\tilde{\Phi}$ which is heavily sensitive to the initial condition on $\tilde{\Phi}\left(\tilde{\Phi}\left(\eta=-10^{6}\right)\right)$ and less sensitive on the other initial condition on $\tilde{\Phi}^{\prime}$. Consequently in this case the perturbation evolution equations do not have large amplitudes as one takes $\tilde{\Phi}^{\prime} \rightarrow 1$. This fact becomes apparent from the plots presented in Fig. 16 and Fig. 17 where in the two plots $\tilde{\Phi}$ remains zero but $\tilde{\Phi}^{\prime}=1$. In this plots the initial conditions for background evolutions, for the symmetric and asymmetric bounces respectively, remain the same as those used for the radiation induced bounces. The wave number of the perturbation mode is $k=10^{-12}$.

The other important point regarding the evolution of the perturbations in the matter induced bounce case is related to presence of the discontinuous jump 
in $\Phi$ in the symmetric bounce background. In the radiation induced symmetric bounce backgrounds this jump was not observed because the amplitude of the discontinuity was much less than the numerical value of $\Phi$ or $\Psi$. In the matter induced bounce the amplitude of $\Phi$ is much less (compared to the amplitude of $\Phi$ in radiation induced bounce) and now the amplitude of the discontinuity shows up. Interestingly in the present case it appears that $\Psi$ is continuous in all the cases. Actually $\Psi$ also has discontinuities at points where $F^{\prime}=0$ but the amplitude of the discontinuity is much less than the value of $\Psi$ and consequently the discontinuities remain hidden.

\section{Discussion and Conclusion}

As mentioned in the introduction of this article there are several papers on cosmological bounce in the framework of GR and some on HD gravity as well. In the works with HD gravity, mainly the bouncing conditions with various forms of $f(R)$ were discussed. A thorough analysis of the whole bouncing mechanism in $f(R)$ theories did not come into sight of the present authors. More over the previous works on cosmological bounce in $f(R)$ theories were done in the Jordan frame itself where interpretation of the dynamics becomes too complicated because of the HD terms involving the Ricci scalar $R$. In the present article our attempt has been to understand the bouncing cosmologies in $f(R)$ theories by studying the corresponding cosmological behavior in the Einstein frame where the theory of gravity is guided by general relativity. Although this prescription is not foolproof as the corresponding theory in the Einstein frame can come with a scalar field whose potential is not bounded from below, but still one can safely use the Einstein frame description of the bouncing phenomenon because before the scalar field starts to role over to the infinite negative depth of the potential the HD theory of gravity itself becomes ineffective.

In this article we started with the general bouncing conditions in the Jordan frame and then focussed on FRW spacetimes which have flat spatial hypersurfaces. A very general proof of the sufficient conditions for the possibility of cosmological bounces, in the absence of any hydrodynamic matter, in such kind of spacetimes is presented in subsection 2.2. In this article we have worked out the full Einstein frame formulation of the cosmological bounce. The Jordan frame and Einstein frame relationships for $f(R)$ theories for inflationary cosmologies 
were known for a long time. But the present case is different because unlike inflationary cosmology, where there is an accelerated expansion of the universe in both the conformally connected frames, here the two frames may show completely different behaviors. We have always assumed that the physical frame is the Jordan frame and so this divergent behaviors do not pose a paradoxical result.

Specifically, the present article deals with the issue of studying bouncing cosmologies in quadratic $f(R)$ theory of gravity where the background FRW line element has zero spatial curvature. The bounces in these cosmologies are interesting because the description of the bouncing phenomena is very different in the Jordan frame and the Einstein frame. If there are cosmological bounces in the Jordan frame there cannot be any analogous bounce in the Einstein frame as shown in subsection 3.2. In the Einstein frame the bouncing regime corresponds to an expanding phase followed by a contracting phase. The change over time from an expanding to a contracting phase depends on the values of the scalar field and its first derivative with respect to the time variable in the Einstein frame. Whereas for a FRW spacetime with non flat spatial hypersurface one may get simultaneous bounces in both the conformally related frames only when the curvature of the spatial hypersurface of the FRW spacetime is positive. In this article we have worked out the explicit nature of the bounces for an universe filled with radiation. From the dynamics of the system in the Einstein frame we could predict about the symmetries of the bouncing phenomenon.

The other important issue discussed in this article is related to the theory of cosmological perturbations in the bouncing universe whose dynamics is guided by a $f(R)$ theory of gravity in the Jordan frame. In this article we have only worked with the scalar sector of the perturbations. Like the background analysis in this case also we first write down the perturbation equations in the Einstein frame, where the analysis becomes relatively simple because the two Bardeen potentials are identical. In this article we derive the main evolution equation for the Bardeen potential in the Einstein frame where the background cosmological evolution takes place in presence of a hydrodynamic fluid and a scalar field. We have assumed that there is only one barotropic fluid in the Jordan frame such that the hydrodynamic perturbations in both the conformally related frames remain adiabatic. The main equation specifying the evolution of the perturbations in the Einstein frame is interesting because from its structure one can see that both the second order time derivative and the Laplacian of the Bardeen potential is multiplied by the square of the speed of sound. Consequently, if the cosmo- 
logical bounce occurs in presence of dust then the evolution of the perturbation equations turn out to be a first order differential equation in conformal time. The main evolution equation of the perturbations reduces to a previously well known evolution equation of the Bardeen potential in the absence of any hydrodynamic fluid. The only difficulty with the present method is related to the fact that the Einstein frame evolution of the perturbation may not give the complete evolution of the Jordan frame perturbation evolution as the Jordan frame potentials may diverge at one instant of time during the bounce. It was shown that this is an artefact of the transformation relations which connect the scalar perturbation potentials in the two conformal frames. This difficulty does not limit the scope of Einstein frame calculation, of the perturbations, as the divergence of the Jordan frame perturbations is purely a local phenomenon happening at an instant of time and the Jordan frame potentials are well behaved throughout the bouncing regime except at that particular time instant.

The way $c_{s}^{2}$ appears in Eq. (65) is in general different from the way it appears in conventional evolution equation of the Bardeen potential in the presence of hydrodynamic matter, where $c_{s}^{2}$ only multiplies the Laplacian operator acting on the Bardeen potential and there is no $c_{s}^{2}$ factor before the second order time derivative of the potential. But it can be verified from the form of Eq. (65) that precisely the positions of the square of the sound velocity there makes the evolution equation correspond to the well known evolution equation of the Bardeen potential in absence of hydrodynamic matter and in the presence of a scalar field, as found in Ref. [35], in appropriate limits.

In this article we did not get the scope to discuss the very important issue of tensor perturbations in the bouncing universe model with $f(R)$ gravity. We presented a numerical solution for the scalar perturbations $\Phi$ and $\Psi$ in the last section which shows that the perturbations remain practically constant during the bouncing period. The topic of tensor perturbations and quantization of the spacetime fluctuations will be addressed in a future publication.

\section{References}

[1] P. A. R. Ade et al. [BICEP2 Collaboration], arXiv:1403.3985 [astroph.CO].

[2] A. Riotto, hep-ph/0210162. 
[3] A. D. Linde, Phys. Scripta T 36, 30 (1991).

[4] R. H. Brandenberger and J. Martin, Mod. Phys. Lett. A 16, 999 (2001) arXiv:astro-ph/0005432.

[5] J. Martin and R. H. Brandenberger, Phys. Rev. D 63, 123501 (2001) arXiv:hep-th/0005209].

[6] R. H. Brandenberger, S. E. Joras and J. Martin, Phys. Rev. D 66, 083514 (2002) arXiv:hep-th/0112122].

[7] J. Martin and R. H. Brandenberger, Phys. Rev. D 65, 103514 (2002)|arXiv:hep-th/0201189].

[8] R. H. Brandenberger and J. Martin, Class. Quant. Grav. 30, 113001 (2013)[arXiv:1211.6753 [astro-ph.CO]].

[9] Z. -G. Liu, Z. -K. Guo and Y. -S. Piao, Phys. Rev. D 88, 063539 (2013) [arXiv:1304.6527 [astro-ph.CO]]

[10] Y. -S. Piao, B. Feng and X. -m. Zhang, Phys. Rev. D 69, 103520 (2004) arXiv:[hep-th/0310206].

[11] Y. -F. Cai, T. -t. Qiu, R. Brandenberger and X. -m. Zhang, Phys. Rev. D 80, 023511 (2009)[arXiv:0810.4677 [hep-th]].

[12] Y. -F. Cai, W. Xue, R. Brandenberger and X. -m. Zhang, JCAP 0906, 037 (2009)[arXiv:0903.4938 [hep-th]].

[13] Y. -F. Cai, D. A. Easson and R. Brandenberger, JCAP 1208, 020 (2012)[arXiv:1206.2382 [hep-th]].

[14] Y. -F. Cai, E. McDonough, F. Duplessis and R. H. Brandenberger, JCAP 1310, 024 (2013)[arXiv:1305.5259 [hep-th]].

[15] K. Bhattacharya, Y. -F. Cai and S. Das, Phys. Rev. D 87, no. 8, 083511 (2013)[arXiv:1301.0661 [hep-th]].

[16] Y. -F. Cai, S. -H. Chen, J. B. Dent, S. Dutta and E. N. Saridakis, Class. Quant. Grav. 28, 215011 (2011) [arXiv:1104.4349 [astro-ph.CO]]. 
[17] Y. -F. Cai and E. N. Saridakis, JCAP 0910, 020 (2009)] [arXiv:0906.1789 [hep-th]].

[18] T. Qiu, X. Gao and E. N. Saridakis, scale-invariant perturbations," Phys. Rev. D 88, no. 4, 043525 (2013) [arXiv:1303.2372 [astro-ph.CO]].

[19] K. Bamba, A. N. Makarenko, A. N. Myagky, S. 'i. Nojiri and S. D. Odintsov, JCAP01(2014)008|arXiv:1309.3748 [hep-th]].

[20] M. Novello and S. E. P. Bergliaffa, Phys. Rept. 463, 127 (2008)[arXiv:0802.1634 [astro-ph]].

[21] S. Carloni, P. K. S. Dunsby and D. M. Solomons, Class. Quant. Grav. 23, 1913 (2006) [arXiv:gr-qc/0510130].

[22] J. D. Barrow and A. C. Ottewill, J. Phys. A 16, 2757 (1983).

[23] S. 'i. Nojiri and S. D. Odintsov, Phys. Rev. D 68, 123512 (2003) [hepth/0307288].

[24] S. M. Carroll, V. Duvvuri, M. Trodden and M. S. Turner, Phys. Rev. D 70, 043528 (2004) [arXiv:astro-ph/0306438].

[25] S. M. Carroll, A. De Felice, V. Duvvuri, D. A. Easson, M. Trodden and M. S. Turner, Phys. Rev. D 71, 063513 (2005)||arXiv:astro-ph/0410031].

[26] L. Amendola, R. Gannouji, D. Polarski and S. Tsujikawa, Phys. Rev. D 75, 083504 (2007) [gr-qc/0612180].

[27] S. 'i. Nojiri and S. D. Odintsov, eConf C 0602061, 06 (2006) [Int. J. Geom. Meth. Mod. Phys. 4, 115 (2007)] [hep-th/0601213].

[28] J. A. R. Cembranos, Phys. Rev. Lett. 102, 141301 (2009) arXiv:0809.1653 [hep-ph]].

[29] S. 'i. Nojiri and S. D. Odintsov, Phys. Rept. 505, 59 (2011)[arXiv:1011.0544 [gr-qc]].

[30] T. P. Sotiriou and V. Faraoni, Rev. Mod. Phys. 82, 451 (2010)[arXiv:0805.1726 [gr-qc]]. 
[31] A. De Felice and S. Tsujikawa, Living Rev. Rel. 13, 3 (2010) [arXiv:1002.4928 [gr-qc]].

[32] T. Clifton, Class. Quant. Grav. 24, 5073 (2007) [gr-qc/0703126 [GR-QC]].

[33] D. Wands, Class. Quant. Grav. 11, 269 (1994) [gr-qc/9307034].

[34] A. A. Starobinsky, Phys. Lett. B 91, 99 (1980).

[35] J. Martin and P. Peter, Phys. Rev. D 68, 103517 (2003) arXiv:hepth/0307077].

[36] R. Utiyama and B. S. DeWitt, J. Math. Phys. 3, 608 (1962).

[37] J. D. Barrow and S. Cotsakis, Phys. Lett. B 214, 515 (1988).

[38] V. Faraoni and E. Gunzig, Int. J. Theor. Phys. 38, 217 (1999) astroph/9910176.

[39] S. Capozziello, S. Nojiri, S. D. Odintsov and A. Troisi, Phys. Lett. B 639, 135 (2006) [astro-ph/0604431].

[40] B. Whitt, Phys. Lett. B 145, 176 (1984).

[41] J. Matsumoto, Phys. Rev. D 87, 104002 (2013) [arXiv:1303.6828 [hep-th]],

[42] D. Bertacca, N. Bartolo and S. Matarrese, JCAP 1208, 021 (2012) [arXiv:1109.2082 [astro-ph.CO]].

[43] V. F. Mukhanov, H. A. Feldman and R. H. Brandenberger, Phys. Rept. 215, 203 (1992).

[44] S. Tsujikawa, R. Gannouji, B. Moraes and D. Polarski, Phys. Rev. D 80, 084044 (2009) [arXiv:0908.2669 [astro-ph.CO]]. 